\title{
Remarks on the static dipole-dipole potential at large distances
}

\author{
Matteo Giordano* \\ Institute for Nuclear Research of the Hungarian Academy of Sciences, \\ Bem tér 18/c, H-4026 Debrecen, Hungary \\ Enrico Meggiolaro \\ Dipartimento di Fisica, Università di Pisa, and INFN, Sezione di Pisa, \\ Largo Pontecorvo 3, I-56127 Pisa, Italy \\ (Received 18 August 2015; published 5 November 2015)
}

\begin{abstract}
We determine the large-distance behavior of the static dipole-dipole potential for a wide class of gauge theories on nonperturbative grounds, exploiting only general properties of the theory. In the case of QCD, we recover the known results in the regime of small dipole sizes and discuss recent nonperturbative calculations. Moreover, we discuss the case of pure-gauge theories and compare our prediction with the available lattice results.
\end{abstract}

DOI: 10.1103/PhysRevD.92.096007

PACS numbers: 11.15.-q, 11.15.Tk

\section{INTRODUCTION}

The potential between two static colorless dipoles is the simplest example of interaction between color-neutral objects that can be studied in the framework of nonAbelian gauge-field theories. The main physical application of this quantity is in the study of the interaction between quarkonia, i.e., mesons made of heavy quarks, which can be treated as static colorless dipoles in a first approximation. From the theoretical point of view, the study of the static dipole-dipole potential poses a nontrivial challenge. Indeed, as one is typically interested in its largedistance behavior to describe the interaction between quarkonia, the interesting properties of the potential are mainly affected by the nonperturbative behavior of the underlying theory, namely QCD. Another complication stems from the fact that the mathematical objects relevant to the theoretical study of the dipole-dipole potential are nonlocal operators, namely Wilson loops.

Calculations of the static dipole-dipole potential available in the literature [1-5] deal with the regime of small dipole sizes in $S U\left(N_{c}\right)$ gauge theories, mostly using perturbative techniques. Even in this somewhat simpler framework, the determination of the static potential requires a careful treatment of color interactions within the dipoles, and of the nonlocality of the Wilson loop, in order to avoid the apparent divergence of the potential. This requires a partial resummation of the perturbative series $[1,2]$, or equivalently a representation of the static dipoles in terms of a series of local operators, in the spirit of the operator product expansion [3-5]. For short interdipole distances $b$, larger than the dipole size $r$ but smaller than the typical hadronic scale, $r \ll b \lesssim 1 \mathrm{fm}$, one can reliably

giordano@atomki.mta.hu enrico.meggiolaro@unipi.it apply perturbation theory to obtain an estimate of the potential, which behaves as $V_{d d} \sim 1 / b^{7}$ [1-5]. At large distances, instead, one has to supplement the perturbative description of the small dipoles with nonpertubative techniques, like the chiral Lagrangians used in Ref. [5]. The leading behavior for $b \gg 1 \mathrm{fm}$ is related to the two-pion threshold and was found to be of the form $V_{d d} \sim e^{-2 m_{\pi} b} / b^{\frac{5}{2}}[4,5]$.

In this paper we want to study the static dipole-dipole potential in a purely nonperturbative setting, starting from the definition in terms of a certain Wilson-loop correlation function and using only general properties of the theory, namely its symmetries and its spectrum, to derive the asymptotic large-distance behavior. The basic idea is to insert a complete set of states between the Wilson loops in the relevant correlation function and relate the large-distance behavior of the potential to the spectrum of the theory.

There are several motivations behind this work. First of all, the fully general results for the dipole-dipole potential derived in this paper provide nontrivial benchmarks for approximate nonperturbative approaches to QCD, like the anti-de Sitter/QCD (AdS/QCD) correspondence or the instanton liquid model (ILM), and to gauge theories in general. In particular, we confirm the previous calculations of Refs. [4,5] and provide a fully nonperturbative definition of the various numerical factors entering $V_{d d}$. We also compare our results to the recent determinations of Refs. [6,7], based on AdS/QCD and on the ILM, respectively. Moreover, since our results apply to a generic gauge theory (with a mass gap), it is possible to obtain information on the interaction of color-neutral states in various theoretically interesting limits, like the isospin limit or the quenched limit, and to establish how sensitive it is to these "deformations" of QCD.

The plan of the paper is the following. After setting the notation in Sec. II, in Sec. III we express the static 
dipole-dipole potential in terms of a sum over a complete set of states. In Sec. IV we study the behavior of the potential at asymptotically large distances, focussing in particular on pure $S U\left(N_{c}\right)$ gauge theory and on gauge theories with light fermions (which include QCD). Finally, in Sec. V we draw our conclusions. Most of the technical details are reported in Appendixes A, B, C, and D.

\section{NOTATION}

In this section we briefly summarize the important points concerning Wilson loops and concerning the sum over a complete set of states, mainly to set the notation.

\section{A. Wilson-loop operators}

In the functional-integral formalism, the Minkowskian Wilson loop $\mathcal{W}_{M}[\mathcal{C}]$ is defined as follows,

$$
\mathcal{W}_{M}[\mathcal{C}]=\frac{1}{N_{c}} \operatorname{trP} \exp \left\{-i g \oint_{\mathcal{C}} A_{\mu}(X) d X^{\mu}\right\},
$$

for a general path $\mathcal{C}$, where $\mathrm{P}$ denotes path ordering ${ }^{1}$ and $A_{\mu}$ are (Minkowskian, Hermitian) non-Abelian gauge fields, taking values in the $N_{c}$-dimensional defining representation of the algebra of the gauge group. The case we have in mind is that of gauge group $S U\left(N_{c}\right)$, but our formalism extends immediately to any subgroup of the unitary groups. In the operator formalism, the Minkowskian Wilson-loop operator reads $[8,9]$

$$
\hat{\mathcal{W}}_{M}[\mathcal{C}]=\frac{1}{N_{c}} \operatorname{trTP} \exp \left\{-i g \oint_{\mathcal{C}} \hat{A}_{\mu}(X) d X^{\mu}\right\},
$$

where $\mathrm{T}$ denotes time ordering of the (Hermitian) nonAbelian gauge-field operators $\hat{A}_{\mu}(X)=e^{i \hat{H} X^{0}} \hat{A}_{\mu}(0, \vec{X}) e^{-i \hat{H} X^{0}}$, where $\hat{H}$ is the Hamiltonian operator. In this paper we will be concerned only with rectangular paths. In Minkowski space, we will denote by $\mathcal{C}_{M}\left(z_{M}, R_{M}, T\right)$ the paths running along the contour of the rectangles $\mathcal{R}_{M}(\sigma, \tau)$,

$\mathcal{R}_{M}(\sigma, \tau)=z_{M}+R_{M} \sigma+T u_{M} \tau, \quad \sigma, \tau \in\left[-\frac{1}{2}, \frac{1}{2}\right]$,

where

$u_{M}=\left(0,1, \overrightarrow{0}_{\perp}\right), \quad R_{M}=\left(r_{\|}, 0, \vec{r}_{\perp}\right), \quad z_{M}=\left(b_{\|}, 0, \vec{b}_{\perp}\right)$.

Notice that here $T$ does not correspond to the time extension of the loop, which is $\left|r_{\|}\right|$instead. For the corresponding Wilson loops (at $z_{M}=0$ ), we will use the following notation:

\footnotetext{
${ }^{1}$ Larger path times appear on the left.
}

$$
\begin{aligned}
& \mathcal{W}_{M}^{(T)}\left(r_{\|}, \vec{r}_{\perp}\right)=\mathcal{W}_{M}\left[\mathcal{C}_{M}\left(0, R_{M}, T\right)\right], \\
& \hat{\mathcal{W}}_{M}^{(T)}\left(r_{\|}, \vec{r}_{\perp}\right)=\hat{\mathcal{W}}_{M}\left[\mathcal{C}_{M}\left(0, R_{M}, T\right)\right] .
\end{aligned}
$$

The Euclidean Wilson loop for a general Euclidean path $\mathcal{C}$, denoted by $\mathcal{W}_{E}[\mathcal{C}]$ in the functional-integral formalism, and by $\hat{\mathcal{W}}_{E}[\mathcal{C}]$ in the operator formalism, is defined exactly as in Eqs. (1) and (2), except that the fields and the scalar product are now Euclidean, and time ordering is with respect to Euclidean "time," which is here the fourth Euclidean coordinate. Explicitly,

$$
\mathcal{W}_{E}[\mathcal{C}]=\frac{1}{N_{c}} \operatorname{trP} \exp \left\{-i g \oint_{\mathcal{C}} A_{E \mu}\left(X_{E}\right) d X_{E \mu}\right\}
$$

in the functional-integral formalism, and

$$
\hat{\mathcal{W}}_{E}[\mathcal{C}]=\frac{1}{N_{c}} \operatorname{trTP} \exp \left\{-i g \oint_{\mathcal{C}} \hat{A}_{E \mu}\left(X_{E}\right) d X_{E \mu}\right\}
$$

in the operator formalism, where $\hat{A}_{E 4}\left(X_{E}\right) \equiv e^{\hat{H} X_{E 4}}(-i) \times$ $\hat{A}_{0}\left(0, \vec{X}_{E}\right) e^{-\hat{H} X_{E 4}}$ and $\hat{A}_{E i}\left(X_{E}\right) \equiv e^{\hat{H} X_{E 4}} \hat{A}_{i}\left(0, \vec{X}_{E}\right) e^{-\hat{H} X_{E 4}}$, $i=1,2,3$ (these operator relations must be understood in the "weak" sense; i.e., they hold for matrix elements of the operators). The Euclidean rectangular paths analogous to those defined in Eq. (3) will be denoted by $\mathcal{C}_{E}\left(z_{E}, R_{E}, T\right)$ and run along the contour of the rectangles $\mathcal{R}_{E}(\sigma, \tau)$ in Euclidean space,

$\mathcal{R}_{E}(\sigma, \tau)=z_{E}+R_{E} \sigma+T u_{E} \tau, \quad \sigma, \tau \in\left[-\frac{1}{2}, \frac{1}{2}\right]$,

where

$u_{E}=\left(1, \overrightarrow{0}_{\perp}, 0\right), \quad R_{E}=\left(0, \vec{r}_{\perp}, r_{\|}\right)=(0, \vec{r})$,

$z_{E}=\left(0, \vec{b}_{\perp}, b_{\|}\right)=(0, \vec{b})$.

For the corresponding Wilson loops (at $z_{E}=0$ ), we will use the following notation:

$$
\begin{aligned}
& \mathcal{W}_{E}^{(T)}\left(r_{\|}, \vec{r}_{\perp}\right)=\mathcal{W}_{E}\left[\mathcal{C}_{E}\left(0, R_{E}, T\right)\right], \\
& \hat{\mathcal{W}}_{E}^{(T)}\left(r_{\|}, \vec{r}_{\perp}\right)=\hat{\mathcal{W}}_{E}\left[\mathcal{C}_{E}\left(0, R_{E}, T\right)\right] .
\end{aligned}
$$

The Euclidean and Minkowskian Wilson loops $\hat{\mathcal{W}}_{E}^{(T)}$ and $\hat{\mathcal{W}}_{M}^{(T)}$ can be formally related by analytic continuation. Indeed, the gauge fields in the Euclidean and Minkowskian Wilson loop appear only in the combinations $\hat{A}_{E \mu}\left(X_{E}\right) d X_{E \mu}$ and $\hat{A}_{\mu}(X) d X^{\mu}$, respectively, which are formally related as follows:

$$
\hat{A}_{E \mu}\left(X_{E}\right) d X_{E \mu}=\left.\hat{A}_{\mu}(X) d X^{\mu}\right|_{r_{\| \rightarrow-i r_{\|}}} .
$$


It then follows that

$$
\hat{\mathcal{W}}_{E}^{(T)}\left(r_{\|}, \vec{r}_{\perp}\right)=\hat{\mathcal{W}}_{M}^{(T)}\left(-i r_{\|}, \vec{r}_{\perp}\right),
$$

again to be understood in the weak sense.

At a certain stage of the calculation, we will need Euclidean Wilson-loop operators running along the same paths $\mathcal{C}_{E}\left(0, R_{E}, T\right)$ appearing in Eq. (10) but corresponding to a different choice of the Euclidean time direction, i.e., obeying a different time ordering. These operators will be denoted by

$$
\begin{aligned}
& \hat{\mathcal{W}}_{E *}^{(T)}\left(r_{\|}, \vec{r}_{\perp}\right) \\
& \quad=\frac{1}{N_{c}} \operatorname{trT}_{1} \mathrm{P} \exp \left\{-i g \oint_{\mathcal{C}_{E}\left(0, R_{E}, T\right)} \hat{A}_{E \mu}\left(X_{E}\right) d X_{E \mu}\right\},
\end{aligned}
$$

where $\mathrm{T}_{1}$ denotes time ordering along direction 1, i.e., (for $T \rightarrow \infty$ ) along the "long" side of the loops.

Let us finally notice that time and path ordering can be disentangled using the continuous-product representation for the path-ordered exponential. In the Minkowskian case, Eq. (2), parametrizing the path as $X(\lambda)$, with $\lambda \in[0,1]$ and $X(0)=X(1)$, one has

$$
\hat{\mathcal{W}}_{M}[\mathcal{C}]=\lim _{N \rightarrow \infty} \sum_{j_{1}, \ldots, j_{N}=1}^{N_{c}} \mathrm{~T}\left\{\prod_{k=0}^{N-1} \hat{w}_{j_{k} j_{k-1}}^{(N)}(k)\right\},
$$

where $j_{k}$ are group indices,

$$
\begin{aligned}
\hat{w}^{(N)}(k) & =\mathbf{1}-\frac{i g}{N} \hat{A}_{\mu}\left(X_{k}\right) \dot{X}_{k}^{\mu}, \quad X_{k}=X\left(\frac{1}{N}\left(k+\frac{1}{2}\right)\right), \\
\dot{X}_{k} & =\frac{d X}{d \lambda}\left(\frac{1}{N}\left(k+\frac{1}{2}\right)\right),
\end{aligned}
$$

with 1 the group identity, and $\hat{w}_{j_{k} j_{k-1}}^{(N)}(k)$ in Eq. (14) are ordered according to $X_{k}^{0}$. Similar representations hold for the Euclidean Wilson-loop operators defined in Eqs. (7) and (13).

\section{B. Complete set of states}

The approach followed in this paper to determine the large-distance behavior of the static dipole-dipole potential is based on the insertion of a complete set of states in a certain Wilson-loop correlation function. We use the complete set of asymptotic "in" states, characterized by their particle content, and by the momenta and third component of the spins of the particles. We define here the setup in full generality, so that the results obtained in this paper can be applied to a wide class of gauge theories.

Let the spectrum of asymptotic states contain $n_{\mathrm{sp}}$ different species of stable particles, characterized by their mass $m_{(s)}$ and spin $s_{(s)}$, with $s \in\left\{1, \ldots, n_{\mathrm{sp}}\right\}$. The particle content of a state is specified by the string $\alpha=$ $\left\{N_{1}, N_{2}, \ldots, N_{n_{\mathrm{sp}}}\right\}$ of the occupation numbers $N_{s}=N_{s}(\alpha)$. For the vacuum $N_{s}=0 \forall s$, we use the notation $\alpha=\emptyset$. Particles are labelled by a double index $i_{s}$, taking values in the index space $S=\left\{i_{s} \mid i_{s} \in \mathbb{N}, s=1, \ldots, n_{\mathrm{sp}}\right\}$. For a given particle content $\alpha$, indices run over the set

$$
S_{\alpha}=\left\{i_{s} \in S \mid 1 \leq i_{s} \leq N_{s}(\alpha), N_{s}(\alpha) \neq 0\right\} ;
$$

the total number of particles is $\mathcal{N}_{\alpha}=\sum_{s} N_{s}(\alpha)$. The momenta, $\vec{p}_{i_{s}}$, and the third component of the spins, $s_{3 i_{s}}$, of all the particles in a state are denoted collectively as $\Omega_{S_{\alpha}}$, where for a general $A \subseteq S$

$$
\Omega_{A}=\left\{\left(\vec{p}_{i_{s}}, s_{3 i_{s}}\right) \mid i_{s} \in A\right\} .
$$

A state is completely specified by $\alpha$ and $\Omega_{S_{\alpha}}$ and will be denoted as follows:

$$
\left.\left|\Omega_{S_{\alpha}}\right\rangle \equiv \mid \cup_{i_{s} \in S_{\alpha}}\left\{\vec{p}_{i_{s}}, s_{3 i_{s}}\right\} ; \text { in }\right\rangle,
$$

where the right-hand side stands for the in state with the appropriate particle content. Such a state transforms under translations and Lorentz transformations as the properly (anti)symmetrized tensor product of the corresponding one-particle states and obeys the usual relativistic normalization. For off-shell momenta, we denote by $\tilde{\Omega}_{A}=\left\{\left(p_{i_{s}}, s_{3 i_{s}}\right) \mid i_{s} \in A\right\}$ the collection of four-momenta and spins. The total energy of a state is denoted as $E\left(\Omega_{S_{\alpha}}\right)$, where for any $A \subseteq S$

$$
E\left(\Omega_{A}\right)=\sum_{i_{s} \in A} \varepsilon_{i_{s}}, \quad \varepsilon_{i_{s}}=\sqrt{\vec{p}_{i_{s}}^{2}+m_{(s)}^{2}} .
$$

Finally, completeness is expressed as

$$
1=\sum_{\alpha} \frac{1}{\prod_{s} N_{s}(\alpha) !} \int d \Omega_{S_{\alpha}}\left|\Omega_{S_{\alpha}}\right\rangle\left\langle\Omega_{S_{\alpha}}\right|,
$$

where for any $A \subseteq S$

$$
\int d \Omega_{A}=\int \prod_{i_{s} \in A}\left[\frac{d^{3} p_{i_{s}}}{(2 \pi)^{3} 2 \varepsilon_{i_{s}}} \sum_{s_{3 i_{s}}=-s_{(s)}}^{s_{(s)}}\right] .
$$

In the following we will also use the notation

$$
\left\langle\left\langle f\left(\Omega_{A}\right)\right\rangle\right\rangle_{\Omega_{A} ; b}=\int d \Omega_{A} e^{-b E\left(\Omega_{A}\right)} f\left(\Omega_{A}\right) .
$$




\section{DIPOLE-DIPOLE POTENTIAL FROM A SUM OVER STATES}

The potential $V_{d d}$ between two static dipoles of size $\vec{r}_{1,2}$, with centers separated by $\vec{b}$, is obtained from the correlation function of two rectangular $T \times\left|\vec{r}_{1,2}\right|$ Euclidean Wilson loops, in the limit of large $T$,

$$
e^{-T V_{d d}} \underset{T \rightarrow \infty}{=} \frac{\left\langle\mathcal{W}_{1}^{(T)} \mathcal{W}_{2}^{(T)}\right\rangle_{E}}{\left\langle\mathcal{W}_{1}^{(T)}\right\rangle_{E}\left\langle\mathcal{W}_{2}^{(T)}\right\rangle_{E}},
$$

where $\mathcal{W}_{1,2}^{(T)}=\mathcal{W}_{E}\left[\mathcal{C}_{1,2}\right]$ for properly chosen paths $\mathcal{C}_{1,2}$ [see Eq. (6)] and $\langle\ldots\rangle_{E}$ denotes the expectation value in the Euclidean functional-integral sense. Without referring to a specific Euclidean coordinate system (for reasons that will be apparent shortly), the Wilson-loop configuration can be described as follows. The size and the relative orientation of the "short" sides of the loops and of the separation between their centers coincide with the size and the relative orientation of $\vec{r}_{1}, \vec{r}_{2}$, and $\vec{b}$. The long sides of the two loops have length $T$, lie in the orthogonal complement of the vector subspace determined by $\vec{r}_{1}, \vec{r}_{2}$, and $\vec{b}$, and are parallel. In a nutshell, our approach to the determination of the large-distance behavior of the dipole-dipole potential consists of going over to the operator formalism and inserting a complete set of states between the loops. Before setting up the calculation in full detail, let us briefly discuss the potentially confusing issue of Euclidean time.

Usually, the long sides of the loops are taken to be parallel to the Euclidean time direction, so that the loops describe the evolution of the dipoles over an amount $T$ of Euclidean time, which is eventually taken to infinity. However, the notion of Euclidean time is well defined only after setting up the Hamiltonian formulation of the theory, while in the Lagrangian formulation employed in the functional-integral formalism, the direction of time can be chosen arbitrarily, thanks to the $O(4)$ invariance of the Euclidean theory. In our approach we exploit this arbitrariness, and we use two different choices of time at different stages of the calculation. For our purposes, it is convenient at first to take time along the separation between the centers of the loops. In this way, after going over to the operator formalism, one can extract the large-distance behavior of the loop-loop correlator in the usual way, by inserting a complete set of states between the Wilson-loop operators. Schematically,

$$
\begin{aligned}
\left\langle\mathcal{W}_{1}^{(T)} \mathcal{W}_{2}^{(T)}\right\rangle_{E}= & \sum_{n} e^{-E_{n}|\vec{b}|}\left\langle 0\left|\hat{\mathcal{W}}_{E}^{(T)}\left(r_{1 \|}, \vec{r}_{1 \perp}\right)\right| n\right\rangle \\
& \times\left\langle n\left|\hat{\mathcal{W}}_{E}^{(T)}\left(r_{2 \|}, \vec{r}_{2 \perp}\right)\right| 0\right\rangle,
\end{aligned}
$$

where $\mathcal{W}_{E}^{(T)}\left(r_{\|}, \vec{r}_{\perp}\right)$ denotes a Wilson loop centered at the origin, spanning a temporal interval of size $\left|r_{\|}\right|$, and with a long spatial side of size $T$ [for more details see Eqs. (8)-(10) and below in this section], and a caret denotes the corresponding operator. Here $|n\rangle$ denotes an energy eigenstate. The large-distance behavior of the correlator is then obtained from the contributions of the lightest states to Eq. (24). To determine the dipole-dipole potential, one has to further take the limit $T \rightarrow \infty$, which affects the Wilsonloop matrix elements appearing in Eq. (24). As we show below in Sec. III A, these matrix elements can be related to the correlation function (again in the sense of the Euclidean functional integral) of a Wilson loop with appropriate Euclidean interpolating fields, corresponding to the particles appearing in the state $|n\rangle$. To study these correlation functions, it is useful to go over again to the operator formalism, but with choosing now the time direction along the long side of the loop,

$$
\begin{aligned}
\left\langle 0\left|\hat{\mathcal{W}}_{E}^{(T)}\left(r_{\|}, \vec{r}_{\perp}\right)\right| n\right\rangle & \rightarrow\left\langle\mathcal{W}_{E}^{(T)}\left(r_{\|}, \vec{r}_{\perp}\right) \prod_{i} \phi_{E i}\left(x_{i}\right)\right\rangle_{E} \\
& =\left\langle 0\left|\mathrm{~T}_{1}\left\{\hat{\mathcal{W}}_{E *}^{(T)}\left(r_{\|}, \vec{r}_{\perp}\right) \prod_{i} \hat{\phi}_{E i}\left(x_{i}\right)\right\}\right| 0\right\rangle,
\end{aligned}
$$

where $\phi_{E i}\left(x_{i}\right)$ are the Euclidean interpolating fields and $\mathrm{T}_{1}$ denotes time ordering of the operators along the direction of the long side of the loop. Here the Wilson-loop operator is denoted by $\hat{\mathcal{W}}_{E *}^{(T)}$, to make it explicit that a different time ordering is used [see Eq. (13)]; although $\hat{\mathcal{W}}_{E}^{(T)}$ and $\hat{\mathcal{W}}_{E *}^{(T)}$ correspond to the same Euclidean path, they are in effect different operators. From the representation Eq. (25), we can then establish the relevant properties of the matrix element in the large- $T$ limit, and by taking $T \rightarrow \infty$, we can finally derive the dipole-dipole potential at large distances. We want to stress the fact that it is perfectly legitimate to use different choices for the time direction to recast the same (functional-integral) correlation function in the operator formalism in different ways, in order to study different aspects of said correlation function, as long as these choices are used consistently. In our case, different choices for the time direction are made in the study of different correlation functions, namely the loop-loop [Eq. (24)] and the loop-interpolating fields [Eq. (25)] correlation functions, so that no inconsistency can arise. We also want to remark that the physical, Minkowskian time plays no role in our calculation, which, starting from Eq. (23), can in principle be performed entirely in Euclidean space. ${ }^{2}$

Let us now return to Eq. (23) and proceed in a more detailed fashion. As we have said above, the $O(4)$ invariance of the Euclidean theory allows us to choose

\footnotetext{
${ }^{2}$ The Minkowskian Wilson loops used in Sec. III A have to be regarded simply as a useful mathematical device; those loops have in fact no relation with the physical process of static dipoles evolving over a large physical time.
} 


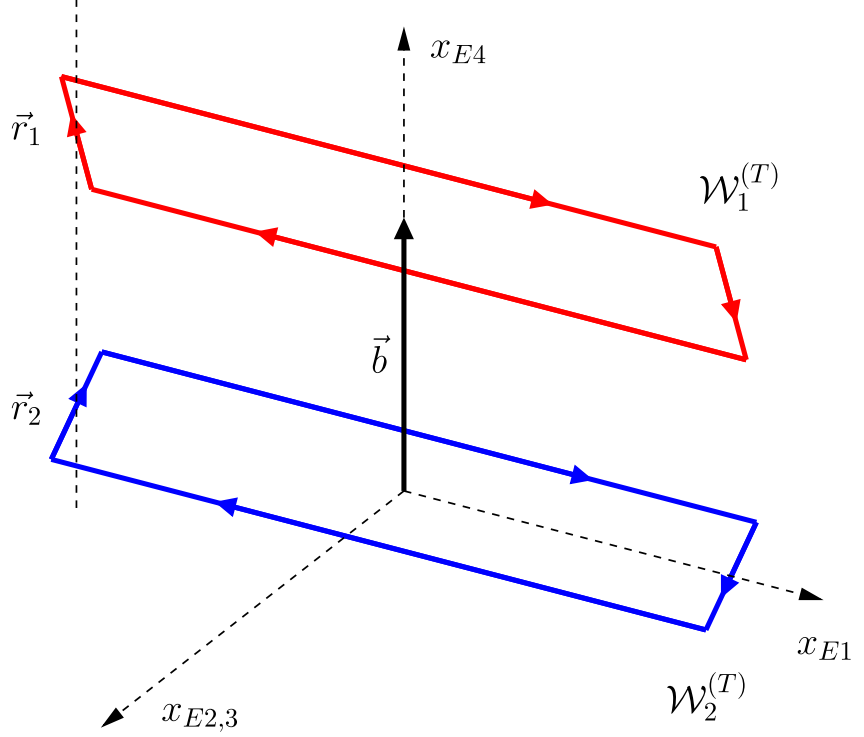

FIG. 1 (color online). The relevant Euclidean Wilson loops.

freely the global orientation of the Wilson-loop configuration. For our purposes, it is convenient to choose $\mathcal{C}_{1,2}$ as follows (see Fig. 1),

$$
\mathcal{C}_{1}=\mathcal{C}_{E}\left(z_{E}, R_{E 1}, T\right), \quad \mathcal{C}_{2}=\mathcal{C}_{E}\left(0, R_{E 2}, T\right),
$$

where the paths $\mathcal{C}_{E}\left(z_{E}, R_{E}, T\right)$ have been defined in Eq. (8), and

$$
\begin{aligned}
R_{E 1,2} & =\left(0, \vec{r}_{1,2 \perp}, r_{1,2 \|}\right)=\left(0, \vec{r}_{1,2}\right), \\
z_{E} & =\left(0, \vec{b}_{\perp}, b_{\|}\right)=(0, \vec{b}) .
\end{aligned}
$$

The Euclidean $O(4)$ invariance further allows us to set $\vec{b}_{\perp}=0$ and $b_{\|}=|\vec{b}| \geq 0$ with no loss of generality. We can thus work in this coordinate frame and write $V_{d d}=V_{d d}\left(b ; r_{1 \|}, \vec{r}_{1 \perp}, r_{2 \|}, \vec{r}_{2 \perp}\right)$, with $b \equiv|\vec{b}|$, so that

$$
\begin{aligned}
\mathcal{G}^{(T)}\left(b ; r_{1 \|}, \vec{r}_{1 \perp}, r_{2 \|}, \vec{r}_{2 \perp}\right) & \equiv \frac{\left\langle\mathcal{W}_{1}^{(T)} \mathcal{W}_{2}^{(T)}\right\rangle_{E}}{\left\langle\mathcal{W}_{1}^{(T)}\right\rangle_{E}\left\langle\mathcal{W}_{2}^{(T)}\right\rangle_{E}} \\
& =e^{-T V_{d d}\left(b ; r_{1 \|}, \vec{r}_{1 \perp}, r_{2 \|}, \vec{r}_{2 \perp}\right)+o(T)} .
\end{aligned}
$$

In the operator formalism, this correlation function reads

$\mathcal{G}^{(T)}\left(b ; r_{1 \|}, \vec{r}_{1 \perp}, r_{2 \|}, \vec{r}_{2 \perp}\right)=\frac{\left\langle 0\left|\mathrm{~T}\left\{\hat{\mathcal{W}}_{1}^{(T)} \hat{\mathcal{W}}_{2}^{(T)}\right\}\right| 0\right\rangle}{\left\langle 0\left|\hat{\mathcal{W}}_{1}^{(T)}\right| 0\right\rangle\left\langle 0\left|\hat{\mathcal{W}}_{2}^{(T)}\right| 0\right\rangle}$,

where $\hat{\mathcal{W}}_{1,2}^{(T)}=\hat{\mathcal{W}}_{E}\left[\mathcal{C}_{1,2}\right]$ [see Eq. (7)]. For loops that do not overlap in the "temporal" direction, i.e., for $b>\left|r_{1 \|}\right|+\left|r_{2 \|}\right|$, the T-ordering sign can be omitted, and so one can insert a complete set of states between the loops. Since in this paper we are interested in the asymptotic large-distance behavior of the potential, we will restrict to this case, without loss of generality. Exploiting time-translation invariance, we can write

$$
\begin{aligned}
\mathcal{G}^{(T)} & \left(b ; r_{1 \|}, \vec{r}_{1 \perp}, r_{2 \|}, \vec{r}_{2 \perp}\right) \\
& =\sum_{\alpha} \frac{1}{\prod_{s} N_{s}(\alpha) !} G_{S_{\alpha}}^{(T)}\left(b ; r_{1 \|}, \vec{r}_{1 \perp}, r_{2 \|}, \vec{r}_{2 \perp}\right),
\end{aligned}
$$

where

$$
\begin{aligned}
& G_{S_{\alpha}}^{(T)}\left(b ; r_{1 \|}, \vec{r}_{1 \perp}, r_{2 \|}, \vec{r}_{2 \perp}\right) \\
& =\left\langle\left\langle M^{(T)}\left(\Omega_{S_{\alpha}} ; r_{1 \|}, \vec{r}_{1 \perp}\right) \bar{M}^{(T)}\left(\Omega_{S_{\alpha}} ; r_{2 \|}, \vec{r}_{2 \perp}\right)\right\rangle\right\rangle_{\Omega_{S_{\alpha}} ; b} \\
& =\int d \Omega_{S_{\alpha}} e^{-b E\left(\Omega_{S_{\alpha}}\right)} M^{(T)}\left(\Omega_{S_{\alpha}} ; r_{1 \|}, \vec{r}_{1 \perp}\right) \bar{M}^{(T)}\left(\Omega_{S_{\alpha}} ; r_{2 \|}, \vec{r}_{2 \perp}\right),
\end{aligned}
$$

and we have denoted as follows the relevant Wilson-loop matrix elements,

$$
\begin{aligned}
& M^{(T)}\left(\Omega_{S_{\alpha}} ; r_{\|}, \vec{r}_{\perp}\right) \equiv \frac{\left\langle 0\left|\hat{\mathcal{W}}_{E}^{(T)}\left(r_{\|}, \vec{r}_{\perp}\right)\right| \Omega_{S_{\alpha}}\right\rangle}{\left\langle 0\left|\hat{\mathcal{W}}_{E}^{(T)}\left(r_{\|}, \vec{r}_{\perp}\right)\right| 0\right\rangle}, \\
& \bar{M}^{(T)}\left(\Omega_{S_{\alpha}} ; r_{\|}, \vec{r}_{\perp}\right) \equiv \frac{\left\langle\Omega_{S_{\alpha}}\left|\hat{\mathcal{W}}_{E}^{(T)}\left(r_{\|}, \vec{r}_{\perp}\right)\right| 0\right\rangle}{\left\langle 0\left|\hat{\mathcal{W}}_{E}^{(T)}\left(r_{\|}, \vec{r}_{\perp}\right)\right| 0\right\rangle},
\end{aligned}
$$

where $\hat{\mathcal{W}}_{E}^{(T)}\left(r_{\|}, \vec{r}_{\perp}\right)$ is computed on the path $\mathcal{C}_{E}\left(0, R_{E}, T\right)$ and has been defined in Eq. (10). Notice that for the vacuum state $G_{S_{\emptyset}}^{(T)}=1$. The two quantities $M^{(T)}$ and $\bar{M}^{(T)}$ can be treated at once by noticing that under Hermitian conjugation

$$
\left[\hat{\mathcal{W}}_{E}^{(T)}\left(r_{\|}, \vec{r}_{\perp}\right)\right]^{\dagger}=\hat{\mathcal{W}}_{E}^{(T)}\left(r_{\|},-\vec{r}_{\perp}\right)
$$

and so it is straightforward to show that

$$
\bar{M}^{(T)}\left(\Omega_{S_{\alpha}} ; r_{\|}, \vec{r}_{\perp}\right)=\left[M^{(T)}\left(\Omega_{S_{\alpha}} ; r_{\|},-\vec{r}_{\perp}\right)\right]^{*} .
$$

In the remainder of this section, we show how the expression Eq. (30) for the Wilson-loop correlator exponentiates to the form given in Eq. (28), with the right $T$-dependence in the large- $T$ limit. The strategy we pursue is the following. We first derive, in Sec. III A, a Euclidean Lehmann-Symanzik-Zimmermann (LSZ) [10,11] representation for the matrix elements, Eq. (32), and from this we obtain, in Sec. III B, a decomposition of the matrix elements in connected components, with each component describing, loosely speaking, the interaction of an isolated subset of particles with the loop. This decomposition allows us to prove the exponentiation of Eq. (30) and finally to establish that the correlator exhibits the correct 
dependence on $T$, in Sec. III C, where the final expression for the dipole-dipole potential is also reported. ${ }^{3}$

\section{A. Euclidean LSZ representation for the matrix elements}

The relevant Euclidean matrix elements $M^{(T)}\left(\Omega_{S_{\alpha}}\right.$; $\left.r_{\|}, \vec{r}_{\perp}\right)$ are related to the analogous matrix elements for the Minkowskian Wilson loop,

$$
M_{M}^{(T)}\left(\Omega_{S_{\alpha}} ; r_{\|}, \vec{r}_{\perp}\right) \equiv \frac{\left\langle 0\left|\hat{\mathcal{W}}_{M}^{(T)}\left(r_{\|}, \vec{r}_{\perp}\right)\right| \Omega_{S_{\alpha}}\right\rangle}{\left\langle 0\left|\hat{\mathcal{W}}_{M}^{(T)}\left(r_{\|}, \vec{r}_{\perp}\right)\right| 0\right\rangle},
$$

by means of analytic continuation [see Eq. (12)],

$$
M^{(T)}\left(\Omega_{S_{\alpha}} ; r_{\|}, \vec{r}_{\perp}\right)=M_{M}^{(T)}\left(\Omega_{S_{\alpha}} ;-i r_{\|}, \vec{r}_{\perp}\right) .
$$

Although the physical quantities entering the dipole-dipole potential are the Euclidean matrix elements $M^{(T)}$, in order to recast them into a LSZ-like expression, it is convenient to work initially with $M_{M}^{(T)}$. The quantity $M_{M}^{(T)}$ admits in fact a straightforward LSZ reduction [10,11], which can be written in the following compact form, ${ }^{4}$

$$
\begin{aligned}
M_{M}^{(T)}\left(\Omega_{S_{\alpha}} ; r_{\|}, \vec{r}_{\perp}\right)= & \operatorname{Lim}_{S_{\alpha}} \Pi\left(\tilde{\Omega}_{S_{\alpha}}\right) \\
& \times L_{M}^{(T)}\left(P_{S_{\alpha}}^{0}, \vec{P}_{S_{\alpha}} ; r_{\|}, \vec{r}_{\perp}\right), \\
L_{M}^{(T)}\left(P_{S_{\alpha}}^{0}, \vec{P}_{S_{\alpha}} ; r_{\|}, \vec{r}_{\perp}\right) \equiv & \int d X_{S_{\alpha}} e^{-i P_{S_{\alpha}} \cdot X_{S_{\alpha}}} \\
& \times \mathcal{C}_{M}^{(T)}\left(X_{S_{\alpha}}^{0}, \vec{X}_{S_{\alpha}} ; r_{\|}, \vec{r}_{\perp}\right),
\end{aligned}
$$

where $\mathcal{C}_{M}^{(T)}$ and $\Pi$ are defined as follows:

\footnotetext{
${ }^{3}$ We notice, incidentally, that the exponentiation of Eq. (30) could be formally obtained in a straightforward way by means of the moments-cumulant theorem. However, this would tell us nothing about the properties of the exponent, so that we could not prove that the correlator has the right $T$-dependence.

${ }^{4}$ The derivation of Eqs. (37) and (38) follows the usual LSZ procedure, the only nontrivial point being the definition of a time-ordered product involving local fields and the nonlocal Wilson-loop operator. This can, however, be easily obtained by using the continuous-product representation of the Wilson loop, Eq. (14), which allows us to write$$
\mathrm{T}\left\{\hat{\mathcal{W}}_{M}[\mathcal{C}] \prod_{i} \hat{\phi}_{i}\left(x_{i}\right)\right\}=\lim _{N \rightarrow \infty} \sum_{j_{1}, \ldots, j_{N}=1}^{N_{c}} \mathrm{~T}\left\{\prod_{k=0}^{N-1} \hat{w}_{j_{k} j_{k-1}}^{(N)}(k) \prod_{i} \hat{\phi}_{i}\left(x_{i}\right)\right\},
$$

for a general path $\mathcal{C}$ and for a general set of local fields $\hat{\phi}_{i}\left(x_{i}\right)$.

$$
\begin{aligned}
\mathcal{C}_{M}^{(T)}\left(X_{S_{\alpha}}^{0}, \vec{X}_{S_{\alpha}} ; r_{\|}, \vec{r}_{\perp}\right) & \\
\equiv & \frac{\left\langle 0\left|\mathrm{~T}\left\{\hat{\mathcal{W}}_{M}^{(T)}\left(r_{\|}, \vec{r}_{\perp}\right) \prod_{i_{s} \in S_{\alpha}} \hat{\Phi}^{(s)}\left(x_{i_{s}}^{0}, \vec{x}_{i_{s}}\right)\right\}\right| 0\right\rangle}{\left\langle 0\left|\hat{\mathcal{W}}_{M}^{(T)}\left(r_{\|}, \vec{r}_{\perp}\right)\right| 0\right\rangle} \\
& =\frac{\left\langle\mathcal{W}_{M}^{(T)}\left(r_{\|}, \vec{r}_{\perp}\right) \prod_{i_{s} \in S_{\alpha}} \Phi^{(s)}\left(x_{i_{s}}^{0}, \vec{x}_{i_{s}}\right)\right\rangle_{M}}{\left\langle\mathcal{W}_{M}^{(T)}\left(r_{\|}, \vec{r}_{\perp}\right)\right\rangle_{M}}, \\
\Pi\left(\tilde{\Omega}_{S_{\alpha}}\right) & \equiv \prod_{i_{s} \in S_{\alpha}} \pi^{(s)}\left(p_{i_{s}}, s_{3 i_{s}}\right) .
\end{aligned}
$$

Here we have introduced some notation that we now explain. $\Pi$ denotes the product of the "projectors" on the appropriate particle poles and spin components: for example, for a scalar particle of mass $m, \pi^{(0)}(p)=p^{2}-m^{2}$; for a spin- $-\frac{1}{2}$ fermion, $\pi^{\left(\frac{1}{2}\right)}\left(p, s_{3}\right)=(\not p-m) u_{s_{3}}(\vec{p})=$ $\left(p^{2}-m^{2}\right)(\not p+m)^{-1} u_{s_{3}}(\vec{p})$; and so on. Both $L_{M}^{(T)}$ and $\Pi$ (may) carry Lorentz indices, appropriately contracted in the product, and are first evaluated off shell; the on-shell limit, denoted with

$$
\operatorname{Lim}_{S_{\alpha}}=\prod_{i_{s} \in S_{\alpha}} \lim _{p_{i_{s}}^{2} \rightarrow m_{(s)}^{2}},
$$

is taken after computing the product. The operators $\hat{\Phi}^{(s)}\left(x_{i_{s}}^{0}, \vec{x}_{i_{s}}\right)$ are the appropriate local interpolating fields for particles of type $s$, normalized to have free-field oneparticle matrix elements, i.e., the renormalization constants required in the LSZ formulas have been absorbed in their definition, so that $\hat{\Phi}^{(s)}$ are renormalized fields. We have denoted collectively with $P_{S_{\alpha}}=\left(P_{S_{\alpha}}^{0}, \vec{P}_{S_{\alpha}}\right)$ the fourmomenta of the particles and the temporal and spatial components thereof. A similar collective notation, $X_{S_{\alpha}}=\left(X_{S_{\alpha}}^{0}, \vec{X}_{S_{\alpha}}\right)$, has been used for the coordinates of the local operators and for the corresponding integration measure, $d X_{S_{\alpha}}$. In the following, when there is no need to distinguish between temporal and spatial components, we do not write them as separate arguments. Moreover, $P_{S_{\alpha}} \cdot X_{S_{\alpha}}=\sum_{i_{s} \in S_{\alpha}} p_{i_{s}} u_{i_{s}}^{\mu}$. Finally, in the third line of Eq. (38), we have used the functional-integral representation for time-ordered vacuum expectation values, denoting with $\langle\ldots\rangle_{M}$ the expectation value in the sense of the Minkowskian functional integral.

The next step is to Wick rotate $L_{M}$ to Euclidean space. By means of a simple change of variables, one shows that

$$
\begin{aligned}
& L_{M}^{(T)}\left(\xi^{-1} P_{S_{\alpha}}^{0}, \vec{P}_{S_{\alpha}} ; \xi r_{\|}, \vec{r}_{\perp}\right) \\
& \quad=\xi^{\mathcal{N}_{\alpha}} \int d X_{S_{\alpha}} e^{-i P_{S_{\alpha}} \cdot X_{S_{\alpha}}} \mathcal{C}_{M}^{(T)}\left(\xi X_{S_{\alpha}}^{0}, \vec{X}_{S_{\alpha}} ; \xi r_{\|}, \vec{r}_{\perp}\right) .
\end{aligned}
$$

By sending $\xi \rightarrow e^{-i \frac{\pi}{2}}$ we then obtain 


$$
\begin{aligned}
& L_{M}^{(T)}\left(e^{i \frac{\pi}{2}} P_{S_{\alpha}}^{0}, \vec{P}_{S_{\alpha}} ; e^{-i \frac{\pi}{2}} r_{\|}, \vec{r}_{\perp}\right) \\
& \quad=(-i)^{\mathcal{N}_{\alpha}} L_{E}^{(T)}\left(-P_{S_{\alpha}}^{0}, \vec{P}_{S_{\alpha}} ; r_{\|}, \vec{r}_{\perp}\right),
\end{aligned}
$$

where we have introduced the Euclidean quantity

$$
\begin{aligned}
& L_{E}^{(T)}\left(P_{E S_{\alpha} 4}, \vec{P}_{E S_{\alpha}} ; r_{\|}, \vec{r}_{\perp}\right) \\
& \equiv \int d X_{E S_{\alpha}} e^{i P_{E S_{\alpha}} \cdot X_{E S_{\alpha}}} \mathcal{C}_{E}^{(T)}\left(\vec{X}_{E S_{\alpha}}, X_{E S_{\alpha} 4}, ; r_{\|}, \vec{r}_{\perp}\right), \\
& \mathcal{C}_{E}^{(T)}\left(\vec{X}_{E S_{\alpha}}, X_{E S_{\alpha} 4}, ; r_{\|}, \vec{r}_{\perp}\right) \\
& \equiv \frac{\left\langle 0\left|\mathrm{~T}\left\{\hat{\mathcal{W}}_{E}^{(T)}\left(r_{\|}, \vec{r}_{\perp}\right) \prod_{i_{s} \in S_{\alpha}} \hat{\Phi}_{E}^{(s)}\left(\vec{x}_{E i_{s}}, x_{E i_{s} 4}\right)\right\}\right| 0\right\rangle}{\left\langle 0\left|\hat{\mathcal{W}}_{E}^{(T)}\left(r_{\|}, \vec{r}_{\perp}\right)\right| 0\right\rangle} \\
& =\frac{\left\langle\mathcal{W}_{E}^{(T)}\left(r_{\|}, \vec{r}_{\perp}\right) \prod_{i_{s} \in S_{\alpha}} \Phi_{E}^{(s)}\left(\vec{x}_{E i_{s}}, x_{E i_{s} 4}\right)\right\rangle_{E}}{\left\langle\mathcal{W}_{E}^{(T)}\left(r_{\|}, \vec{r}_{\perp}\right)\right\rangle_{E}},
\end{aligned}
$$

where $P_{E S_{\alpha}}=\left(\vec{P}_{E S_{\alpha}}, P_{E S_{\alpha} 4}\right)$ denotes collectively the Euclidean four-momenta $p_{E i_{s}}, X_{E S_{\alpha}}=\left(\vec{X}_{E S_{\alpha}}, X_{E S_{\alpha} 4}\right)$ the coordinates of the local operators, $d X_{E S_{\alpha}}$ the corresponding integration measure, and $P_{E S_{\alpha}} \cdot X_{E S_{\alpha}}=\sum_{i_{s} \in S_{\alpha}} p_{E i_{s} \mu} x_{E i_{s} \mu}$. The Euclidean Wilson loop $\hat{\mathcal{W}}_{E}^{(T)}$ has been defined in Eq. (7), and $\hat{\Phi}_{E}^{(s)}$ are now the appropriate local functionals of the Euclidean fields. In the last line, we have made contact with the Euclidean functional-integral formalism.

Inverting the analytic-continuation relation, we find ${ }^{5}$

$$
\begin{aligned}
& L_{M}^{(T)}\left(P_{S_{\alpha}}^{0}, \vec{P}_{S_{\alpha}} ; r_{\|}, \vec{r}_{\perp}\right) \\
& \quad=(-i)^{\mathcal{N}_{\alpha}} L_{E}^{(T)}\left(e^{-i \frac{\pi}{2}}\left(-P_{S_{\alpha}}^{0}\right), \vec{P}_{S_{\alpha}} ; e^{i \frac{\pi}{2}} r_{\|}, \vec{r}_{\perp}\right), \\
& L_{M}^{(T)}\left(P_{S_{\alpha}}^{0}, \vec{P}_{S_{\alpha}} ;-i r_{\|}, \vec{r}_{\perp}\right) \\
& \quad=(-i)^{\mathcal{N}_{\alpha}} L_{E}^{(T)}\left(e^{-i \frac{\pi}{2}}\left(-P_{S_{\alpha}}^{0}\right), \vec{P}_{S_{\alpha}} ; r_{\|}, \vec{r}_{\perp}\right) .
\end{aligned}
$$

Summarizing, $M^{(T)}$ is obtained by first computing $L_{E}^{(T)}$ for real arguments, then performing the Wick rotation to obtain $L_{M}^{(T)}$ (with real arguments), taking the momenta on shell and finally analytically continuing $r_{\|} \rightarrow-i r_{\|}$. However, the on-shell projection and the last analytic continuation $r_{\|} \rightarrow-i r_{\|}$should not interfere. If it is so, then

$$
\begin{aligned}
& M^{(T)}\left(\Omega_{S_{\alpha}} ; r_{\|}, \vec{r}_{\perp}\right) \\
& \quad=\operatorname{Lim}_{S_{\alpha}} \Pi\left(\tilde{\Omega}_{S_{\alpha}}\right)(-i)^{\mathcal{N}_{\alpha}} L_{E}^{(T)}\left(e^{-i \frac{\pi}{2}}\left(-P_{S_{\alpha}}^{0}\right), \vec{P}_{S_{\alpha}} ; r_{\|}, \vec{r}_{\perp}\right),
\end{aligned}
$$

\footnotetext{
${ }^{5}$ The notation $e^{-i \frac{\pi}{2}}\left(-P_{S_{\alpha}}^{0}\right)$ indicates that to obtain the correlator at positive (off-shell) energies $p_{i_{s}}^{0}$ one starts from negative $p_{E i_{s} 4}=-p_{i_{s}}^{0}$ and then rotates clockwise in the complex $p_{E i_{s} 4}$ plane.
}

and we can follow a simpler route: after computing $L_{E \alpha}^{(T)}$ for real arguments, we perform the partial Wick rotation $L_{E \alpha}^{(T)}\left(e^{-i \frac{\pi}{2}}\left(-P_{S_{\alpha}}^{0}\right), \vec{P}_{S_{\alpha}} ; r_{\|}, \vec{r}_{\perp}\right)$ and finally take the momenta on shell.

Let us finally notice that the correlator $\mathcal{C}_{E}^{(T)}$ in Eq. (42) is a renormalized quantity. Indeed, we are working with renormalized interpolating fields [see the discussion after Eq. (39)], and moreover the Euclidean Wilson loop enters $\mathcal{C}_{E}^{(T)}$ through the combination $\mathcal{W}_{E}^{(T)} /\left\langle\mathcal{W}_{E}^{(T)}\right\rangle_{E}$, which is a renormalization-invariant quantity since $\mathcal{W}_{E}^{(T)}$ renormalizes multiplicatively $[12,13]$. As a consequence, the matrix elements $M^{(T)}$, Eq. (32) [as well as the Minkowskian matrix elements $M_{M}^{(T)}$, Eq. (35)], are renormalized (and renormalization-invariant) quantities.

\section{B. Cluster decomposition of the matrix elements}

The point in relating $M^{(T)}$ with the purely Euclidean quantity $L_{E}^{(T)}$ is that the latter admits a neat cluster decomposition. Furthermore, as the Euclidean functional integral admits a nonperturbative definition through the lattice discretization, we can perform the formal manipulations rather safely. To compute the correlation function $\mathcal{C}_{E}^{(T)}$, Eq. (42), we can exploit once again the $O(4)$ invariance of the Euclidean theory and choose the time direction as we please. For our purposes, it is convenient to now take time along direction 1, i.e., the direction of the long side of the loop. Reverting to the operator formalism with this choice for the time direction, we write

$$
\begin{aligned}
\mathcal{C}_{E}^{(T)}\left(X_{E S_{\alpha}} ; r_{\|}, \vec{r}_{\perp}\right) & =\frac{\left\langle 0\left|\mathrm{~T}_{1}\left\{\hat{\mathcal{W}}_{E *}^{(T)}\left(r_{\|}, \vec{r}_{\perp}\right) \hat{\mathcal{O}}_{S_{\alpha}}\left(X_{E S_{\alpha}}\right)\right\}\right| 0\right\rangle}{\left\langle 0\left|\hat{\mathcal{W}}_{E *}^{(T)}\left(r_{\|}, \vec{r}_{\perp}\right)\right| 0\right\rangle}, \\
\hat{\mathcal{O}}_{S_{\alpha}}\left(X_{E S_{\alpha}}\right) & \equiv \prod_{i_{s} \in S_{\alpha}} \hat{\Phi}_{E}^{(s)}\left(\vec{x}_{E i_{s}}, x_{E i_{s} 4}\right),
\end{aligned}
$$

where $\hat{\mathcal{W}}_{E *}^{(T)}$ has been defined in Eq. (13) and $\mathrm{T}_{1}$ denotes time ordering along direction 1 . As $\mathcal{C}_{E}^{(T)}$ is gauge invariant, we can work in the temporal gauge where the long sides of the loop are trivial. With this choice of time ordering and in this gauge, the Wilson-loop operator can be expressed in terms of the following Wilson-line operator,

$$
\begin{aligned}
\hat{W}_{E}\left(R_{E}\right) & =\mathrm{P} \exp \left\{-i g \int_{-\frac{1}{2}}^{+\frac{1}{2}} d \lambda \hat{A}_{E \mu}\left(\lambda R_{E}\right) R_{E \mu}\right\}, \\
\hat{W}_{E}\left(-R_{E}\right) & =\hat{W}_{E}\left(R_{E}\right)^{\dagger},
\end{aligned}
$$

where the time-ordering symbol has been dropped, since only gauge fields at $x_{E 1}=0$ appear. In terms of $\hat{W}_{E}$, the Wilson-loop operator reads 


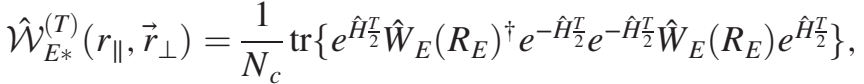

with $\hat{H}$ the Hamiltonian operator. Since we are ultimately interested in the limit $T \rightarrow \infty$, we consider only the case when $T / 2>\left|x_{E i_{s} 1}\right| \forall i_{s} \in S_{\alpha}$. Inserting complete sets of states in the appropriate sector of the theory (namely, that transforming as a pair of color charges in the fundamental and complex conjugate representation located at a distance $R_{E}$ ), we find

$$
\begin{aligned}
& \left\langle 0\left|\mathrm{~T}_{1}\left\{\hat{\mathcal{W}}_{E *}^{(T)}\left(r_{\|}, \vec{r}_{\perp}\right) \hat{\mathcal{O}}_{S_{\alpha}}\left(X_{E S_{\alpha}}\right)\right\}\right| 0\right\rangle \\
& =\sum_{s_{1}, s_{2}} \sum_{i, j} e^{-\frac{T}{2}\left(E_{s_{1}}+E_{s_{2}}\right)}\left\langle R_{E} ; i j \mid s_{1}\right\rangle\left\langle s_{1}\left|\mathrm{~T}_{1}\left\{\hat{\mathcal{O}}_{S_{\alpha}}\left(X_{E S_{\alpha}}\right)\right\}\right| s_{2}\right\rangle \\
& \quad \times\left\langle s_{2} \mid R_{E} ; i j\right\rangle
\end{aligned}
$$

and moreover

$$
\begin{aligned}
& \left\langle 0\left|\hat{\mathcal{W}}_{E *}^{(T)}\left(r_{\|}, \vec{r}_{\perp}\right)\right| 0\right\rangle \\
& \quad=\sum_{s_{1}} \sum_{i, j} e^{-T E_{s_{1}}}\left\langle R_{E} ; i j \mid s_{1}\right\rangle\left\langle s_{1} \mid R_{E} ; i j\right\rangle,
\end{aligned}
$$

where $\left|R_{E} ; i j\right\rangle \equiv\left[\hat{W}_{E}\left(R_{E}\right)\right]_{i j}|0\rangle$ is the "flux-tube" state created by the Wilson line $\hat{W}_{E}\left(R_{E}\right)$. In the limit $T \rightarrow \infty$, the dominant contribution comes from the flux-tube ground state, $s_{1}=s_{2}=g=g\left(R_{E}\right)$ (since there is a gap with the first excited state), and we obtain

$$
\begin{aligned}
\mathcal{C}_{E}\left(X_{E S_{\alpha}} ; r_{\|}, \vec{r}_{\perp}\right) & \equiv \lim _{T \rightarrow \infty} \mathcal{C}_{E}^{(T)}\left(X_{E S_{\alpha}} ; r_{\|}, \vec{r}_{\perp}\right) \\
& =\left\langle g\left|\mathrm{~T}_{1}\left\{\hat{\mathcal{O}}_{S_{\alpha}}\left(X_{E S_{\alpha}}\right)\right\}\right| g\right\rangle .
\end{aligned}
$$

Consider now the case when the interpolating fields cluster into subsets, well separated from each other in the time direction. More precisely, given a partition $\mathcal{A}_{K}\left(S_{\alpha}\right)$ of $S_{\alpha}$ in $K$ parts, $\mathcal{A}_{K}\left(S_{\alpha}\right)=\left\{a_{k}\right\}_{k=1, \ldots, K}$, consider the limit

$\left|x_{E i_{s} 1}-x_{E i_{s}^{\prime} 1}\right| \rightarrow \infty, \quad \forall i_{s} \in a_{k}, \quad \forall i_{s^{\prime}}^{\prime} \in a_{k^{\prime}}, \quad k \neq k^{\prime}$.

By appropriately inserting complete sets of flux-tube states between the subsets of interpolating fields, one can show that in this limit the sums over intermediate states are dominated by the ground state, and so

$$
\begin{aligned}
\mathcal{C}_{E}\left(X_{E S_{\alpha}} ; r_{\|}, \vec{r}_{\perp}\right) & \rightarrow \prod_{k=1}^{K} \mathcal{C}_{E}\left(X_{E a_{k}} ; r_{\|}, \vec{r}_{\perp}\right) \\
& =\prod_{a \in \mathcal{A}_{K}\left(S_{\alpha}\right)} \mathcal{C}_{E}\left(X_{E a} ; r_{\|}, \vec{r}_{\perp}\right) .
\end{aligned}
$$

Let us now perform a decomposition in connected components in the usual way, i.e., defining recursively, for any $T$, and for $A \subseteq S$,

$$
\begin{aligned}
\mathcal{C}_{E}^{(T) \operatorname{conn}}\left(X_{E A} ; r_{\|}, \vec{r}_{\perp}\right) \\
\equiv \mathcal{C}_{E}^{(T)}\left(X_{E A} ; r_{\|}, \vec{r}_{\perp}\right) \\
\quad-\sum_{K} \sum_{\mathcal{A}_{K}(A) \neq\{A\}} \prod_{a \in \mathcal{A}_{K}(A)} \mathcal{C}_{E}^{(T) \operatorname{conn}}\left(X_{E a} ; r_{\|}, \vec{r}_{\perp}\right),
\end{aligned}
$$

where the sum is over all partitions of $A,\{A\}$ is the trivial partition, and $\mathcal{C}_{E}^{(T) \text { conn }}=\mathcal{C}_{E}^{(T)}$ for one-element sets, so that

$$
\begin{aligned}
& \mathcal{C}_{E}^{(T)}\left(X_{E S_{\alpha}} ; r_{\|}, \vec{r}_{\perp}\right) \\
& \quad=\sum_{K} \sum_{\mathcal{A}_{K}\left(S_{\alpha}\right)} \prod_{a \in \mathcal{A}_{K}\left(S_{\alpha}\right)} \mathcal{C}_{E}^{(T) \mathrm{conn}}\left(X_{E a} ; r_{\|}, \vec{r}_{\perp}\right) .
\end{aligned}
$$

In the limit $T \rightarrow \infty$, one has analogously

$$
\begin{aligned}
& \mathcal{C}_{E}\left(X_{E S_{\alpha}} ; r_{\|}, \vec{r}_{\perp}\right) \\
& \quad=\sum_{K} \sum_{\mathcal{A}_{K}\left(S_{\alpha}\right)} \prod_{a \in \mathcal{A}_{K}\left(S_{\alpha}\right)} \mathcal{C}_{E}^{\text {conn }}\left(X_{E a} ; r_{\|}, \vec{r}_{\perp}\right) .
\end{aligned}
$$

In this limit, $\mathcal{C}_{E}$ is translation invariant along the time direction, i.e., direction 1, and so, by construction [see Eq. (53)], each connected component $\mathcal{C}_{E}^{\text {conn }}$ is also similarly invariant under time translations. Moreover, Eq. (52) shows that in the limit $T \rightarrow \infty$ each connected component vanishes when at least one of the interpolating fields is very far from the others in the time direction. Let us make this discussion explicit by writing

$$
\mathcal{C}_{E}^{(T) \operatorname{conn}}\left(X_{E a}\right)=C_{T}\left(t_{a}, \hat{X}_{a}\right),
$$

where $t_{a}=\frac{1}{N_{a}} \sum_{i_{s} \in a} x_{E i_{s} 1}$ is the average time coordinate of the particles in part $a$, with $N_{a}$ the corresponding number of particles, and $\hat{X}_{a}$ denotes collectively all the remaining components of the coordinates. Here we have dropped the dependence on the dipole size for simplicity. As $T \rightarrow \infty$,

$$
\lim _{T \rightarrow \infty} C_{T}\left(t_{a}, \hat{X}_{a}\right)=C\left(\hat{X}_{a}\right)
$$

We can also say something about how this limit is approached. At finite $T, C_{T}\left(t_{a}, \hat{X}_{a}\right)$ is essentially constant for $\left|t_{a}\right| \ll \frac{T}{2}$ and should not change appreciably as long as $\left|t_{a}\right|<\frac{T}{2}-\kappa a_{\text {corr }}$, where $a_{\text {corr }}$ is the so-called "vacuum correlation length" [14-16] and $\kappa$ is some number of order 1 , that depends also on the spread of the temporal components of the positions of the interpolating fields (which again can be at most a few $a_{\text {corr }}$ since we are considering a connected correlation function), but that is independent of $T$ (when $T$ is large enough and only one short edge at a time is relevant to this issue). After a 
transient region of size approximately $2 \kappa a_{\text {corr }}$, the correlator drops essentially to zero when $\left|t_{a}\right|>\frac{T}{2}+\kappa a_{\text {corr }}$. The conclusion is that $C_{T}\left(T \tau_{a}, \hat{X}_{a}\right)$ tends to a constant function over the interval $\tau_{a} \in\left[-\frac{1}{2}, \frac{1}{2}\right]$ : the transient regions in terms of $\tau_{a}$ shrink as $T \rightarrow \infty$, and the slope of the function there diverges. So $C_{T}\left(t_{a}, \hat{X}_{a}\right) \rightarrow \chi\left(\frac{t_{a}}{T}\right) C\left(\hat{X}_{a}\right)$, or more precisely

$$
\lim _{T \rightarrow \infty} C_{T}\left(T \tau_{a}, \hat{X}_{a}\right)=\chi\left(\tau_{a}\right) C\left(\hat{X}_{a}\right),
$$

with $\chi\left(\tau_{a}\right)$ the characteristic function of the interval $\left[-\frac{1}{2}, \frac{1}{2}\right]$.

Cluster decompositions for $L_{E}^{(T)}$ and $M^{(T)}$ can also be written down, in full analogy with Eqs. (53) and (54). Comparing them with the cluster decomposition of $\mathcal{C}_{E}^{(T)}$, one finds

$$
\begin{aligned}
& L_{E}^{(T) \operatorname{conn}}\left(P_{E a} ; r_{\|}, \vec{r}_{\perp}\right) \\
& =\int d X_{E a} e^{i P_{E a} \cdot X_{E a}} \mathcal{C}_{E}^{(T) \operatorname{conn}}\left(X_{E a} ; r_{\|}, \vec{r}_{\perp}\right), \\
& M^{(T) \operatorname{conn}}\left(\Omega_{a} ; r_{\|}, \vec{r}_{\perp}\right) \\
& =\operatorname{Lim}_{a} \prod_{i_{s} \in a}\left[-i \pi^{(s)}\left(\vec{p}_{i_{s}}, s_{3 i_{s}}\right)\right] \\
& \quad \times L_{E}^{(T) \operatorname{conn}}\left(e^{-i \frac{\pi}{2}}\left(-P_{a}^{0}\right), \vec{P}_{a} ; r_{\|}, \vec{r}_{\perp}\right) .
\end{aligned}
$$

Here we have made use of the fact that the on-shell projector is factorized. The connected components of $\bar{M}^{(T)}$ are easily obtained using Eq. (34),

$$
\bar{M}^{(T) \operatorname{conn}}\left(\Omega_{a} ; r_{\|}, \vec{r}_{\perp}\right) \equiv\left[M^{(T) \operatorname{conn}}\left(\Omega_{a} ; r_{\|},-\vec{r}_{\perp}\right)\right]^{*} .
$$

Finally, a similar decomposition can be carried out for the various quantities in the limit $T \rightarrow \infty$.

The time-translation invariance of $\mathcal{C}_{E}^{\text {conn }}$, for a certain part, $a$, in some partition, $\mathcal{A}_{K}$, reflects itself in the appearance of delta functions in $L_{E}^{\text {conn }} \equiv \lim _{T \rightarrow \infty} L_{E}^{(T) \text { conn }}$, imposing the vanishing of the total temporal momentum of the particles in $a$. Furthermore, as $\mathcal{C}_{E}^{\text {conn }}$ vanishes when the time separation between the interpolating fields becomes large [see Eq. (52)], the corresponding integration regions give no contribution to $L_{E}^{\text {conn }}$, and no further delta functions of subsets of temporal momenta can appear. Finally, as the analytic continuation required to obtain the matrix elements $M=\lim _{T \rightarrow \infty} M^{(T)}$ does not involve $p_{E i_{\mathrm{s}} 1}$, these properties are inherited by the connected components $M^{\text {conn }} \equiv$ $\lim _{T \rightarrow \infty} M^{(T) \text { conn }}$, which contain one and the same delta function of the temporal momenta as $L_{E}^{\text {conn }}$. More precisely, for the physically relevant quantity $M^{(T) \text { conn }}$, one can write $M^{(T) \operatorname{conn}}\left(\Omega_{a} ; r_{1 \|}, \vec{r}_{1 \perp}\right)=\int d t_{a} e^{i q_{a} t_{a}} F_{T}\left(t_{a}, P_{a} ; r_{1 \|}, \vec{r}_{1 \perp}\right)$, where $q_{a} \equiv \sum_{i_{s} \in a} p_{i_{s} 1}$, for a certain function $F_{T}$, obtained from $C_{T}$ through integration over $\hat{X}_{a}$, Wick rotation of the momenta, and on-shell projection (see Appendix $\mathrm{C}$ for more details). The important point is that these steps should not change the way the large- $T$ limit is approached; i.e., for large $T$

$$
\begin{aligned}
& \lim _{T \rightarrow \infty} F_{T}\left(T \tau_{a}, P_{a} ; r_{1 \|}, \vec{r}_{1 \perp}\right) \\
& \quad=\chi\left(\tau_{a}\right) \mathcal{M}^{\operatorname{conn}}\left(\Omega_{a} ; r_{1 \|}, \vec{r}_{1 \perp}\right),
\end{aligned}
$$

for a certain $\mathcal{M}^{\text {conn }}$, from which it follows

$$
\begin{aligned}
& \lim _{T \rightarrow \infty} M^{(T) \operatorname{conn}}\left(\Omega_{a} ; r_{1 \|}, \vec{r}_{1 \perp}\right) \\
& \quad=\delta\left(\sum_{i_{s} \in a} p_{i_{s} 1}\right) \mathcal{M}^{\mathrm{conn}}\left(\Omega_{a} ; r_{1 \|}, \vec{r}_{1 \perp}\right) .
\end{aligned}
$$

For the other connected matrix element, $\bar{M}^{(T) \text { conn }}$ [see Eq. (60)], we similarly have

$$
\begin{aligned}
& \lim _{T \rightarrow \infty} \bar{M}^{(T) \operatorname{conn}}\left(\Omega_{a} ; r_{2 \|}, \vec{r}_{2 \perp}\right) \\
& \quad=\delta\left(\sum_{i_{s} \in a} p_{i_{s} 1}\right) \overline{\mathcal{M}}^{\mathrm{conn}}\left(\Omega_{a} ; r_{2 \|}, \vec{r}_{2 \perp}\right) \\
& \overline{\mathcal{M}}^{\operatorname{conn}}\left(\Omega_{a} ; r_{2 \|}, \vec{r}_{2 \perp}\right) \\
& =\left[\mathcal{M}^{\operatorname{conn}}\left(\Omega_{a} ; r_{2 \|},-\vec{r}_{2 \perp}\right)\right]^{*} .
\end{aligned}
$$

\section{Dipole-dipole potential from the Wilson-loop correlator}

The purpose of the analysis of the previous subsection is twofold. On the one hand, the cluster decomposition allows us to write down explicitly the exponential form of the Wilson-loop correlator, Eq. (30). On the other hand, the properties of the connected components in the large- $T$ limit imply that the correct $T$-dependence is obtained.

Let us start from the exponentiation. The decomposition of the matrix elements into connected components is not yet the full story, since what appears in Eq. (30) is the product of the matrix elements $M^{(T)}$ and $\bar{M}^{(T)}$. Substituting the cluster decompositions of $M^{(T)}$ and $\bar{M}^{(T)}$ in Eq. (30), one thus obtains a double sum over partitions. Each pair of partitions $\mathcal{A}_{K}\left(S_{\alpha}\right), \overline{\mathcal{A}}_{\bar{K}}\left(S_{\alpha}\right)$ of $S_{\alpha}$, with $K$ and $\bar{K}$ parts, respectively, i.e., $\mathcal{A}_{K}\left(S_{\alpha}\right)=\left\{a_{k}\right\}_{k=1, \ldots, K}$ and $\overline{\mathcal{A}}_{\bar{K}}\left(S_{\alpha}\right)=$ $\left\{\bar{a}_{\bar{k}}\right\}_{\bar{k}=1, \ldots, \bar{K}}$, can be uniquely rewritten as a partition $\mathcal{F}_{J}\left(S_{\alpha}\right)$ of $S_{\alpha}$ with $J$ parts and a set of irreducible pairs of partitions $\left[\mathcal{A}_{K_{j}}, \overline{\mathcal{A}}_{\bar{K}_{j}}\right]\left(F_{j}\right)$ of the parts $F_{j} \in \mathcal{F}_{J}\left(S_{\alpha}\right)$. By an irreducible pair of partitions we mean that there are no proper subpartitions $\left\{a_{k}^{\prime}\right\}_{k=1, \ldots, K_{j}^{\prime}} \subset \mathcal{A}_{K_{j}}\left(F_{j}\right)$, and $\left\{\bar{a}_{\bar{k}}^{\prime}\right\}_{\bar{k}=1, \ldots, \bar{K}_{j}^{\prime}} \subset \overline{\mathcal{A}}_{\bar{K}_{j}}\left(F_{j}\right)$, such that $\cup_{k} a_{k}^{\prime}=\cup_{\bar{k}} \bar{a}_{\bar{k}}^{\prime}$. Checking a few examples should convince the reader; a formal proof is given in Appendix A. The double sum over partitions can therefore be rewritten as 


$$
\begin{aligned}
\sum_{K} & \sum_{\mathcal{A}_{K}\left(S_{\alpha}\right)} \sum_{\bar{K}} \sum_{\overline{\mathcal{A}}_{\bar{K}}\left(S_{\alpha}\right)} \\
& =\sum_{J} \sum_{\mathcal{F}_{J}\left(S_{\alpha}\right)} \prod_{F \in \mathcal{F}_{J}\left(S_{\alpha}\right)}\left(\sum_{K} \sum_{L} \sum_{\left[\mathcal{A}_{K}, \overline{\mathcal{A}}_{\bar{K}}\right](F)}\right) .
\end{aligned}
$$

Working out the consequences of this fact is a straightforward but lengthy exercise in combinatorics, which is described in detail in Appendix B. Here we report only the final result for the Wilson-loop correlator, which reads

$$
\begin{aligned}
\mathcal{G}^{(T)} & \left(b ; r_{1 \|}, \vec{r}_{1 \perp}, r_{2 \|}, \vec{r}_{2 \perp}\right) \\
& =\exp \left\{\sum_{\alpha \neq \emptyset} \frac{1}{\prod_{s} N_{s}(\alpha) !} \mathcal{Q}_{\alpha}^{(T)}\left(b ; r_{1 \|}, \vec{r}_{1 \perp}, r_{2 \|}, \vec{r}_{2 \perp}\right)\right\},
\end{aligned}
$$

where we have introduced the following quantities:

$$
\begin{aligned}
& \mathcal{Q}_{\alpha}^{(T)}\left(b ; r_{1 \|}, \vec{r}_{1 \perp}, r_{2 \|}, \vec{r}_{2 \perp}\right) \\
& =\sum_{K} \sum_{\bar{K}} \sum_{\left[\mathcal{A}_{K}, \overline{\mathcal{A}}_{\bar{K}}\right]\left(S_{\alpha}\right)}\left\langle\left\langle\prod_{a \in \mathcal{A}_{K}\left(S_{\alpha}\right)} M^{(T) \operatorname{conn}}\left(\Omega_{a} ; r_{1 \|}, \vec{r}_{1 \perp}\right)\right.\right. \\
& \left.\quad \times \prod_{\bar{a} \in \overline{\mathcal{A}}_{\bar{K}}\left(S_{\alpha}\right)} \bar{M}^{(T) \operatorname{conn}}\left(\Omega_{\bar{a}} ; r_{2 \|}, \vec{r}_{2 \perp}\right)\right\rangle_{\Omega_{S_{\alpha}} ; b}
\end{aligned}
$$

Recalling Eq. (28), the dipole-dipole potential reads

$$
\begin{aligned}
V_{d d}\left(b ; r_{1 \|}, \vec{r}_{1 \perp}, r_{2 \|}, \vec{r}_{2 \perp}\right) \\
=-\lim _{T \rightarrow \infty} \frac{1}{T} \sum_{\alpha \neq \emptyset} \frac{1}{\prod_{s} N_{s}(\alpha) !} \mathcal{Q}_{\alpha}^{(T)}\left(b ; r_{1 \|}, \vec{r}_{1 \perp}, r_{2 \|}, \vec{r}_{2 \perp}\right) \\
=\sum_{\alpha \neq \emptyset} \frac{1}{\prod_{s} N_{s}(\alpha) !} \sum_{K} \sum_{\bar{K}} \\
\quad \times \sum_{\left[\mathcal{A}_{K}, \overline{\mathcal{A}}_{\bar{K}}\right]\left(S_{\alpha}\right)} V_{\left[\mathcal{A}_{K}, \overline{\mathcal{A}}_{\bar{K}}\right]\left(S_{\alpha}\right)}\left(b ; r_{1 \|}, \vec{r}_{1 \perp}, r_{2 \|}, \vec{r}_{2 \perp}\right)
\end{aligned}
$$

where $\left[\mathcal{A}_{K}, \overline{\mathcal{A}}_{\bar{K}}\right]\left(S_{\alpha}\right)$ is a pair of irreducible partitions of $S_{\alpha}$, and

$$
\begin{aligned}
& -V_{\left[\mathcal{A}_{K}, \overline{\mathcal{A}}_{\bar{K}}\right]\left(S_{\alpha}\right)}\left(b ; r_{1 \|}, \vec{r}_{1 \perp}, r_{2 \|}, \vec{r}_{2 \perp}\right) \\
& \equiv \lim _{T \rightarrow \infty} \frac{1}{T}\left\langle\left\langle\prod_{a \in \mathcal{A}_{K}\left(S_{\alpha}\right)} M^{(T) \operatorname{conn}}\left(\Omega_{a} ; r_{1 \|}, \vec{r}_{1 \perp}\right)\right.\right. \\
& \left.\left.\quad \times \prod_{\bar{a} \in \overline{\mathcal{A}}_{\bar{K}}\left(S_{\alpha}\right)} \bar{M}^{(T) \operatorname{conn}}\left(\Omega_{\bar{a}} ; r_{2 \|}, \vec{r}_{2 \perp}\right)\right\rangle\right\rangle_{\Omega_{S_{\alpha}} ; b} .
\end{aligned}
$$

The crucial point is now to show that $\mathcal{Q}_{\alpha}^{(T)}$ diverges linearly with $T$. As we have argued in the previous subsection, in the large- $T$ limit, each connected component develops a Dirac delta of the total temporal momenta $q_{a} \equiv \sum_{i_{s} \in a} p_{i_{s} 1}$ and $\bar{q}_{\bar{a}} \equiv \sum_{i_{s} \in \bar{a}} p_{i_{s} 1}$ in each part. In Appendix A we show that, due to the irreducibility of the pair of partitions, only $K+\bar{K}-1 \leq \mathcal{N}_{\alpha}$ out of the $K+\bar{K}$ linear combinations of momenta $q_{a}$ and $\bar{q}_{\bar{a}}$ are independent, the only relation of linear dependence being

$$
\sum_{a \in \mathcal{A}_{K}\left(S_{\alpha}\right)} q_{a}=\sum_{\bar{a} \in \overline{\mathcal{A}}_{\bar{K}}\left(S_{\alpha}\right)} \bar{q}_{\bar{a}}=\sum_{i_{s} \in S_{\alpha}} p_{i_{s} 1} .
$$

In practical terms, this means that in the large- $T$ limit the integral in Eq. (69) is divergent, as one of the $K+\bar{K}$ Dirac deltas of Eqs. (63) and (64) has to be evaluated at zero. However, this also means that the divergence is linear in $T$, so that it gets cancelled by the $1 / T$ factor, and $V_{\left[\mathcal{A}_{K}, \overline{\mathcal{A}}_{\bar{K}}\right]\left(S_{\alpha}\right)}$ is finite. A detailed calculation showing this, which makes use of the large- $T$ behavior of the connected matrix elements, Eq. (62), is reported in Appendix C. Here we quote only the final result,

$$
\begin{aligned}
& -V_{\left[\mathcal{A}_{K}, \overline{\mathcal{A}}_{\bar{K}}\right]\left(S_{\alpha}\right)}\left(b ; r_{1 \|}, \vec{r}_{1 \perp}, r_{2 \|}, \vec{r}_{2 \perp}\right) \\
& =\int d \Omega_{S_{\alpha}} e^{-b E\left(\Omega_{S_{\alpha}}\right)}(2 \pi)^{K+\bar{K}-1} \delta_{\left[\mathcal{A}_{K}, \overline{\mathcal{A}}_{\bar{K}}\right]\left(S_{\alpha}\right)}\left(p_{1}\right) \\
& \quad \times \prod_{a \in \mathcal{A}_{K}\left(S_{\alpha}\right)} \mathcal{M}^{\operatorname{conn}}\left(\Omega_{a} ; r_{1 \|}, \vec{r}_{1 \perp}\right) \\
& \quad \times \prod_{\bar{a} \in \overline{\mathcal{A}}_{\bar{K}}\left(S_{\alpha}\right)} \overline{\mathcal{M}}^{\operatorname{conn}}\left(\Omega_{\bar{a}} ; r_{2 \|}, \vec{r}_{2 \perp}\right), \\
& \delta_{\left[\mathcal{A}_{K}, \overline{\mathcal{A}}_{\bar{K}}\right]\left(S_{\alpha}\right)}\left(p_{1}\right) \\
& \equiv \delta_{\left(\sum_{i_{s} \in S_{\alpha}} p_{i_{s} 1}\right)}^{\circ} \prod_{a \in \mathcal{A}_{K}\left(S_{\alpha}\right)} \delta\left(\sum_{i_{s} \in a} p_{i_{s} 1}\right) \\
& \quad \times \prod_{\bar{a} \in \overline{\mathcal{A}}_{\bar{K}}\left(S_{\alpha}\right)} \delta\left(\sum_{i_{s} \in \bar{a}} p_{i_{s} 1}\right),
\end{aligned}
$$

where the symbol $\circ$ denotes that the product is over all the parts in the partition but one.

The expressions Eqs. (68) and (71) fully encode the static dipole-dipole potential when the dipoles do not overlap in the direction of their separation, i.e., for all $\vec{b}$ and $\vec{r}_{1,2}$ such that $|\vec{b}|>\left|\vec{r}_{1} \cdot \hat{b}\right|+\left|\vec{r}_{2} \cdot \hat{b}\right|$. In the next section, we use them to extract the behavior of the potential at asymptotically large distances.

\section{ASYMPTOTIC BEHAVIOR OF THE POTENTIAL AT LARGE DISTANCE}

At this point it is straightforward to derive the large- $b$ behavior of the potential. From Eq. (71) we see that the $b$-dependence is contained entirely in the factor $e^{-b E\left(\Omega_{S_{\alpha}}\right)}$. However, we still have to perform the phase-space integration. Making the change of variables $\sqrt{b} \vec{p}_{i_{s}}=\vec{q}_{i_{s}}$, we can rewrite Eq. (71) as follows, 


$$
\begin{aligned}
& -V_{\left[\mathcal{A}_{K}, \overline{\mathcal{A}}_{\bar{K}}\right]\left(S_{\alpha}\right)}\left(b ; r_{1 \|}, \vec{r}_{1 \perp}, r_{2 \|}, \vec{r}_{2 \perp}\right) \\
& =b^{-\frac{3 \mathcal{N}_{\alpha}-(K+\bar{K}-1)}{2}} \int d \hat{\Omega}_{S_{\alpha}} e^{-b \sum_{i_{s} \in S_{\alpha}} m_{(s)} \hat{\varepsilon}_{i_{s}}}(2 \pi)^{K+\bar{K}-1} \\
& \quad \times \delta_{\left[\mathcal{A}_{K}, \overline{\mathcal{A}}_{\bar{K}}\right]\left(S_{\alpha}\right)}\left(q_{1}\right) \prod_{a \in \mathcal{A}_{K}\left(S_{\alpha}\right)} \mathcal{M}^{\operatorname{conn}}\left(\frac{1}{\sqrt{b}} \hat{\Omega}_{a} ; r_{1 \|}, \vec{r}_{1 \perp}\right) \\
& \quad \times \prod_{\bar{a} \in \overline{\mathcal{A}}_{\bar{K}}\left(S_{\alpha}\right)} \overline{\mathcal{M}}^{\operatorname{conn}}\left(\frac{1}{\sqrt{b}} \hat{\Omega}_{\bar{a}} ; r_{2 \|}, \vec{r}_{2 \perp}\right),
\end{aligned}
$$

where

$$
\begin{aligned}
\int d \hat{\Omega}_{S_{\alpha}} & \equiv \sum_{\left\{s_{3}\right\}} \prod_{i_{s} \in S_{\alpha}} \int \frac{d^{3} q_{i_{s}}}{(2 \pi)^{3} 2 m_{(s)} \hat{\varepsilon}_{i_{s}}}, \\
\hat{\varepsilon}_{i_{s}} & \equiv \sqrt{1+\frac{\vec{q}_{i_{s}}^{2}}{b m_{(s)}^{2}}},
\end{aligned}
$$

$\sum_{\left\{s_{3}\right\}}$ denotes the sum over the spins of all particles, and we have denoted

$$
\frac{1}{\sqrt{b}} \hat{\Omega}_{A}=\left\{\left(\frac{1}{\sqrt{b}} \vec{q}_{i_{s}}, s_{3 i_{s}}\right) \mid i_{s} \in A\right\}, \quad A \subseteq S .
$$

In the limit of large $b$, we can expand $\hat{\varepsilon}_{i_{s}}$ and the integration measure $d \hat{\Omega}_{S_{\alpha}}$ as follows,

$$
\begin{aligned}
\hat{\varepsilon}_{i_{s}} & =1+\frac{\vec{q}_{i_{s}}^{2}}{2 b m_{(s)}^{2}}+\mathcal{O}\left(b^{-2}\right), \\
d \hat{\Omega}_{S_{\alpha}} & =\prod_{i_{s} \in S_{\alpha}} \frac{d^{3} q_{i_{s}}}{(2 \pi)^{3} 2 m_{(s)}\left(1+\mathcal{O}\left(b^{-1}\right)\right)} \\
& \equiv d q_{S_{\alpha}}\left(1+\mathcal{O}\left(b^{-1}\right)\right),
\end{aligned}
$$

and moreover we can expand the matrix elements around zero momentum,

$$
\begin{aligned}
\frac{1}{\sqrt{b}} \hat{\Omega}_{A} & =\left\{\left(0, s_{3 i_{s}}\right) \mid i_{s} \in A\right\}+\mathcal{O}\left(b^{-\frac{1}{2}}\right) \\
& \equiv \Omega_{A}^{0}+\mathcal{O}\left(b^{-\frac{1}{2}}\right)
\end{aligned}
$$

To leading order we find

$$
\begin{aligned}
& -V_{\left[\mathcal{A}_{K}, \overline{\mathcal{A}}_{\bar{K}}\right]\left(S_{\alpha}\right)}\left(b ; r_{1 \|}, \vec{r}_{1 \perp}, r_{2 \|}, \vec{r}_{2 \perp}\right) \\
& \underset{b \rightarrow \infty}{\rightarrow} b^{-\frac{3 \mathcal{N}_{\alpha}-(K+\bar{K}-1)}{2}} e^{-b \sum_{s} m_{(s)} N_{s}(\alpha)} \mathscr{M}_{\left[\mathcal{A}_{K}, \overline{\mathcal{A}}_{\bar{K}]}\right]\left(S_{\alpha}\right)} \\
& \quad \times \mathcal{M}_{\left[\mathcal{A}_{K}, \overline{\mathcal{A}}_{\bar{K}]}\right]\left(S_{\alpha}\right)}\left(r_{1 \|}, \vec{r}_{1 \perp}, r_{2 \|}, \vec{r}_{2 \perp}\right),
\end{aligned}
$$

where the full $b$-dependence is in the first two factors; $\mathscr{M}_{\left[\mathcal{A}_{K}, \overline{\mathcal{A}}_{\bar{K}}\right]\left(S_{\alpha}\right)}$ is a constant,

$$
\begin{aligned}
\mathscr{M}_{\left[\mathcal{A}_{K}, \overline{\mathcal{A}}_{\bar{K}}\right]\left(S_{\alpha}\right)} & \\
\equiv & \int d q_{S_{\alpha}} e^{-\sum_{i_{s} \in S_{\alpha}} \frac{\bar{q}_{i s}^{2}}{i_{(s)}}}(2 \pi)^{K+\bar{K}-1} \delta_{\left[\mathcal{A}_{K}, \overline{\mathcal{A}}_{\bar{K}}\right]\left(S_{\alpha}\right)}\left(q_{1}\right) \\
= & \frac{1}{2^{\mathcal{N}_{\alpha}}(2 \pi)^{2 \mathcal{N}_{\alpha}-(K+\bar{K}-1)}} \int\left[\prod_{i_{s} \in S_{\alpha}} d q_{i_{s} 1} e^{-\frac{q_{i_{s} 1}^{2}}{2 m_{(s)}}}\right] \\
& \times \delta_{\left[\mathcal{A}_{K}, \overline{\mathcal{A}}_{\bar{K}}\right]\left(S_{\alpha}\right)}\left(q_{1}\right) ;
\end{aligned}
$$

and the dependence on the size and orientation of the dipoles is contained in $\mathcal{M}_{\left[\mathcal{A}_{K}, \overline{\mathcal{A}}_{\bar{K}}\right]\left(S_{\alpha}\right)}$,

$$
\begin{aligned}
& \mathcal{M}_{\left[\mathcal{A}_{K}, \overline{\mathcal{A}}_{\bar{K}}\right]\left(S_{\alpha}\right)}\left(r_{1 \|}, \vec{r}_{1 \perp}, r_{2 \|}, \vec{r}_{2 \perp}\right) \\
& \equiv \sum_{\left\{s_{3}\right\}} \prod_{a \in \mathcal{A}_{K}\left(S_{\alpha}\right)} \mathcal{M}^{\operatorname{conn}}\left(\Omega_{a}^{0} ; r_{1 \|}, \vec{r}_{1 \perp}\right) \\
& \quad \times \prod_{\bar{a} \in \overline{\mathcal{A}}_{\bar{K}}\left(S_{\alpha}\right)} \overline{\mathcal{M}}^{\operatorname{conn}}\left(\Omega_{\bar{a}}^{0} ; r_{2 \|}, \vec{r}_{2 \perp}\right) .
\end{aligned}
$$

Here we are implicitly assuming that the connected matrix elements $\mathcal{M}^{\text {conn }}$ are finite, nonzero quantities at zero momentum. This is expected to be the case for states containing only massive particles. ${ }^{6}$ Notice that the exponent $\gamma=\left[3 \mathcal{N}_{\alpha}-(K+\bar{K}-1)\right] / 2$ of the power-law term in Eqs. (72) and (77) obeys the inequality $\gamma \geq \mathcal{N}_{\alpha} \geq 1$ (see the end of Appendix A), as well as $\gamma \leq\left(3 \mathcal{N}_{\alpha}-1\right) / 2$ since $K$, $\bar{K} \geq 1$, for any (nonvacuum) state.

The leading behavior of the potential is determined by the contributions $V_{\left[\mathcal{A}_{K}, \overline{\mathcal{A}}_{\bar{K}}\right]\left(S_{\alpha}\right)}$ of the lightest states with nonzero Wilson-loop matrix elements, with higher-order contributions being exponentially suppressed. Since the Wilson-loop operator depends only on the gauge fields, it is obviously invariant under any symmetry of the theory acting only on the matter degrees of freedom. This implies a selection rule involving the corresponding quantum numbers, which have to be the same as those of the vacuum in order for the Wilson-loop matrix element to be nonzero. In particular, in the case of QCD, the Wilson loop is insensitive to flavor, and so its matrix elements can be nonzero only for states carrying no flavor quantum numbers, which results in a selection rule for baryon number, electric charge, strangeness, etc., that must all vanish.

For the interesting gauge theories, the lightest particle is typically a spin-zero particle. Indeed, lattice results for $S U\left(N_{c}\right)$ pure-gauge theory indicate that the lightest

\footnotetext{
${ }^{6}$ In the presence of massless particles, they are expected to vanish, in order to cancel the divergence in the phase-space measure. We have verified this explicitly in the simple case of pure $U(1)$ gauge theory, i.e., for free photons. Notice that, in the case discussed in the present paper, the above-mentioned divergence is only apparent and does not require the vanishing of the matrix elements. However, one can easily show that in the case of Wilson loops at nonzero angle $\theta$, considered, e.g., in Ref. [17], there is indeed a logarithmic divergence unless the matrix elements vanish.
} 
"glueball" has quantum numbers $J^{P C}=0^{++}$(see, e.g., Ref. [18]). For theories with $N_{f}$ light fermions, the lightest particles are the $N_{f}^{2}-1$ (pseudo-)Goldstone bosons generated by the spontaneous breaking of the (approximate) chiral $S U\left(N_{f}\right)_{L} \times S U\left(N_{f}\right)_{R}$ symmetry (at least if $N_{f}$ is not too large). This is the case for real-world QCD $\left(N_{c}=3, N_{f}=2\right)$, where the lightest states are the pions (pseudoscalars). For spin-zero particles, it is possible to derive easily further selection rules on parity and charge conjugation. As we show in Appendix D, for a self-conjugate particle with $C$ and $P$ phases $\eta_{C}$ and $\eta_{P}$, nonzero matrix elements are possible only if $\eta_{C}=\eta_{P}=1$.

Let us now discuss in detail a few interesting cases. In QCD, the lightest particles are the three pions, $\pi^{0}$ and $\pi^{ \pm}$, but due to the selection rules on electric charge and on parity, they have vanishing one-particle matrix elements. The lightest state with nonzero matrix element is the one containing two $\pi^{0}$, followed by the state containing a $\pi^{+} \pi^{-}$pair. In both cases there is a single irreducible pair of partitions contributing to the potential, namely the pair of trivial partitions $\left[\mathcal{A}_{1}, \overline{\mathcal{A}}_{1}\right]$, and so denoting with $S_{\pi^{0} \pi^{0}}$ and $S_{\pi^{+} \pi^{-}}$the relevant $S_{\alpha}$, we find from Eq. (78)

$$
\begin{aligned}
\mathscr{M}_{\left[\mathcal{A}_{1}, \overline{\mathcal{A}}_{1}\right]\left(S_{\pi^{0} \pi^{0}}\right)} & =\frac{\sqrt{m_{\pi^{0}}}}{(4 \pi)^{\frac{5}{2}}}, \\
\mathscr{M}_{\left[\mathcal{A}_{1}, \overline{\mathcal{A}}_{1}\right]\left(S_{\pi^{+} \pi^{-}}\right)} & =\frac{\sqrt{m_{\pi^{ \pm}}}}{(4 \pi)^{\frac{5}{2}}} .
\end{aligned}
$$

Due to the very small relative mass difference between the neutral and the charged pions (also when electromagnetic effects are neglected), the $\pi^{+} \pi^{-}$contribution is appreciably suppressed compared to the $\pi^{0} \pi^{0}$ contribution only for distances well beyond the range of the dipole-dipole interaction. Therefore, although strictly speaking it is the $\pi^{0} \pi^{0}$ state that determines the asymptotic behavior of the potential, it is physically more meaningful to treat charged and neutral pions on the same footing. We will then consider the limit of exact isospin symmetry and ignore the small mass difference between $\pi^{0}$ and $\pi^{ \pm}$. In this limit the contributions of the $\pi^{0} \pi^{0}$ state and of the $\pi^{+} \pi^{-}$state are identical, ${ }^{7}$ and so, taking into account the symmetry factor $1 / 2$ for the $\pi^{0} \pi^{0}$ state, we have to leading order $^{8}$

\footnotetext{
${ }^{7}$ As the Wilson loop is flavor blind, the only contributions to the Wilson-loop matrix elements $M^{(T)}$ come from the isosinglet components of these states, which differ only by a sign.

${ }^{8}$ According to the discussion above, in real QCD the strict asymptotic behavior is obtained from Eq. (81) by replacing the factor $3 / 2$ with $1 / 2$ and using the $\pi^{0}$ mass and $\pi^{0} \pi^{0}$ matrix elements.
}

$$
\begin{aligned}
& V_{d d}\left(b ; r_{1 \|}, \vec{r}_{1 \perp}, r_{2 \|}, \vec{r}_{2 \perp}\right) \\
& \underset{b \rightarrow \infty}{\rightarrow} V_{\left[\mathcal{A}_{1}, \overline{\mathcal{A}}_{1}\right]\left(S_{\pi \pi}\right)}\left(b ; r_{1 \|}, \vec{r}_{1 \perp}, r_{2 \|}, \vec{r}_{2 \perp}\right) \\
& \underset{b \rightarrow \infty}{\rightarrow}-\frac{3}{2} \sqrt{m_{\pi}} \frac{e^{-2 m_{\pi} b}}{(4 \pi b)^{\frac{5}{2}}} \mathcal{M}^{\operatorname{conn}}\left(\Omega_{S_{\pi \pi}}^{0} ; r_{1 \|}, \vec{r}_{1 \perp}\right) \\
& \quad \times \overline{\mathcal{M}}^{\operatorname{conn}}\left(\Omega_{S_{\pi \pi}}^{0} ; r_{2 \|}, \vec{r}_{2 \perp}\right) .
\end{aligned}
$$

Due to Eq. (64), the potential is attractive at large distances. ${ }^{9}$ For $N_{f}$ degenerate flavors of quarks $q_{i}$, the relevant states are those with pairs of "pions" $\pi_{i j} \pi_{j i}$, where $\pi_{i j}=q_{i} \bar{q}_{j}$ for $i \neq j$, and $N_{f}-1$ pairs $\pi_{i} \pi_{i}$ with $\pi_{i}$ a combination of $q_{i} \bar{q}_{i}$ (the completely symmetric one is excluded). There are $N_{f}\left(N_{f}-1\right) / 2$ pairs with $i \neq j$, and the $N_{f}-1$ states with two $\pi_{i}$ require a symmetry factor $1 / 2$; the net effect is to replace

$$
\frac{3}{2} \rightarrow \frac{N_{f}\left(N_{f}-1\right)}{2}+\frac{N_{f}-1}{2}=\frac{N_{f}^{2}-1}{2}
$$

in Eq. (81).

The dependence on $b$ and the properties of our result, Eqs. (81) and (82), agree with the findings of Refs. [4,5], which apply in the regime of small dipole sizes. On the other hand, the calculations of Refs. [6,7], via AdS/QCD and in the ILM, respectively, report large-distance behaviors of the form $V_{d d}^{\mathrm{AdS} / \mathrm{QCD}} \sim e^{-M_{X} b} / b^{\frac{3}{2}}$ [see Ref. [6], Eq. (37)] and $V_{d d}^{\mathrm{ILM}} \sim e^{-m_{s} b} / b^{\frac{1}{2}}$ [see Ref. [7], Eq. (64)], where $M_{X}=m_{\rho} \sqrt{17 / 8}$ with $m_{\rho}$ the rho mass, and $m_{s} \sim 350 \mathrm{MeV} .{ }^{10}$ Concerning the ILM result, the mass scale $m_{s}$ is approximately of the right magnitude. ${ }^{11}$ On the other hand, the mass scale $M_{X}$ is clearly much larger than the pion threshold. However, the AdS/QCD

\footnotetext{
${ }^{9}$ More precisely, this is certainly true for small enough dipole sizes if the matrix elements are nonvanishing and analytic in $r_{\|}$ and $\vec{r}_{\perp}$ at zero. If the matrix elements are continuous and never vanish, then this is true for all dipole sizes. Furthermore, notice that Eq. (64) implies that this is true for $r_{2 \|}=r_{1 \|}, \vec{r}_{2 \perp}=-\vec{r}_{1 \perp}$. As we show in Appendix D, $\mathcal{M}^{\text {conn }}\left(\Omega_{S_{\pi \pi}}^{0} ; r_{\|}, \vec{r}_{\perp}\right)$ depends on $\vec{r}_{\perp}$ only through $\vec{r}_{\perp}^{2}$, so this is again true for $r_{2 \|}=r_{1 \|},\left|\vec{r}_{2 \perp}\right|=\left|\vec{r}_{1 \perp}\right|$.

${ }^{10}$ The fractional powers of $b$ look troublesome, since they cannot appear in our general formulas, Eqs. (72) and (77). Indeed, turning around the inequalitites reported after Eq. (79), we have $(2 \gamma+1) / 3 \leq \mathcal{N}_{\alpha} \leq \gamma$, that cannot be satisfied by any integer $\mathcal{N}_{\alpha}$ for $\gamma=\frac{1}{2}, \frac{3}{2}$. However, in our opinion these values are due to small mistakes in the extraction of the asymptotic behavior of the potential. Correcting these mistakes we find $V_{d d}^{\mathrm{AdS} / \mathrm{QCD}} \sim$ $e^{-M_{X} b} / b$, and $V_{d d}^{\mathrm{ILM}} \sim e^{-m_{s} b} / b$, which match the form of oneparticle contributions to the potential.

${ }^{11}$ The mass $m_{s}$ corresponds to a scalar glueball state in the ILM [7], which is stable to leading order in $1 / N_{c}$ but which develops a nonvanishing decay width in higher orders [19], that turns it into a resonant two-pion state; this could explain the "anomalous" power-law correction $b^{-1}$ to the exponential decay in the corrected expression for $V_{d d}^{\mathrm{ILM}}$ reported in footnote 10 .
} 

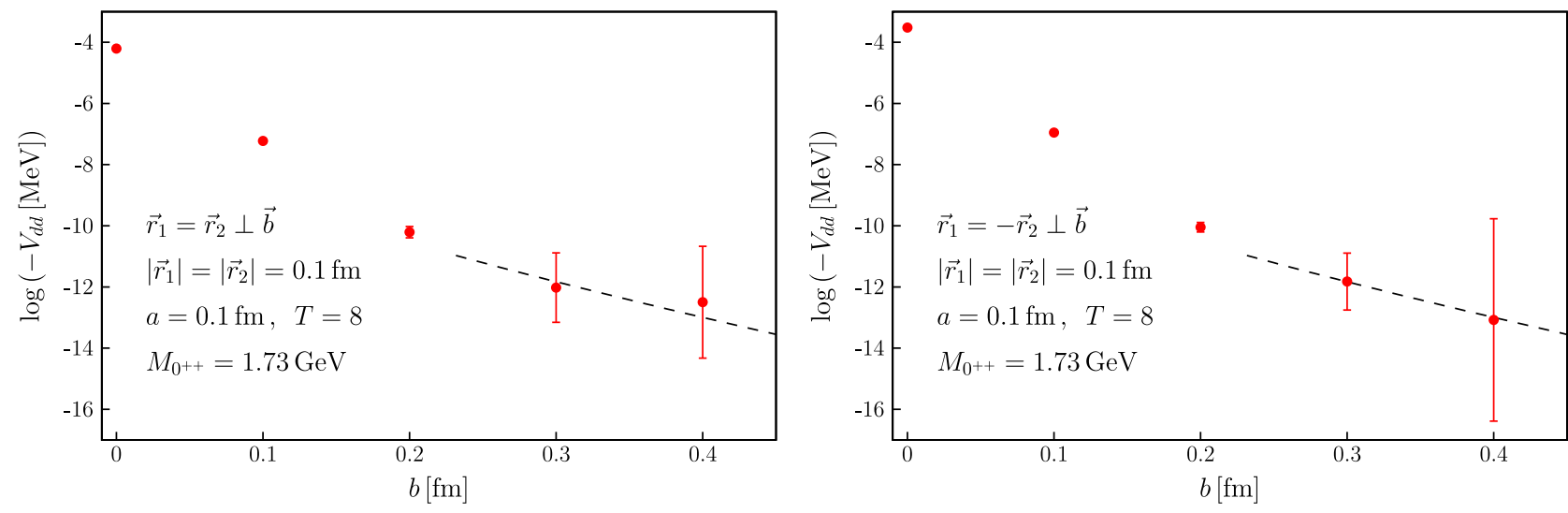

FIG. 2 (color online). Lattice determination of the static dipole-dipole potential in quenched QCD (data are taken from Ref. [22]). Only statistical errors are shown. The dashed line corresponds to the asymptotic behavior, Eq. (84), with the numerical prefactor adjusted to fit the data points at $b=0.3,0.4 \mathrm{fm}$.

correspondence is expected to hold in the large- $N_{c}$ and strong-coupling limits, and so it is perhaps more appropriate to compare this result to the one we have obtained in pure-gauge theory, discussed below. In this case the mass scale $M_{X}$ is of the right order of magnitude, although still quite smaller than the lightest glueball mass at large $N_{c}$ (which is slightly larger than at $N_{c}=3$ [18]; see below). In the estimate one should probably use the quenched value $m_{\rho}^{\mathrm{q}}$ for the rho mass, which, however, does not differ too much from the physical value; using the quenched lattice results of Ref. [20] for quenched pion masses $m_{\pi}^{\mathrm{q}}$ below $m_{\pi}^{\mathrm{q}} \lesssim 400 \mathrm{MeV}$, one has $m_{\rho}^{\mathrm{q}} \simeq 800-900 \mathrm{MeV}$, resulting in $M_{X} \sim 1.2-1.3 \mathrm{GeV}$, i.e., about $30 \%$ below the lightest glueball mass.

In pure $S U\left(N_{c}\right)$ gauge theory, the lightest state contributing to the potential is the one containing a single $0^{++}$ glueball, which we denote by $S_{0^{++}}$. In this case there is obviously a single relevant pair of partitions, and so

$$
\mathscr{M}_{\left[\mathcal{A}_{1}, \overline{\mathcal{A}}_{1}\right]\left(S_{0^{++}}\right)}=\frac{1}{4 \pi},
$$

so that

$$
\begin{aligned}
& V_{d d}\left(b ; r_{1 \|}, \vec{r}_{1 \perp}, r_{2 \|}, \vec{r}_{2 \perp}\right) \\
& \underset{b \rightarrow \infty}{\rightarrow} V_{\left[\mathcal{A}_{1}, \overline{\mathcal{A}}_{1}\right]\left(S_{0^{++}}\right)}\left(b ; r_{1 \|}, \vec{r}_{1 \perp}, r_{2 \|}, \vec{r}_{2 \perp}\right) \\
& \underset{b \rightarrow \infty}{\rightarrow}-\frac{e^{-m_{0^{++}} b}}{4 \pi b} \mathcal{M}^{\operatorname{conn}}\left(\Omega_{S_{0^{++}}^{0}}^{0} ; r_{1 \|}, \vec{r}_{1 \perp}\right) \\
& \quad \times \overline{\mathcal{M}}^{\operatorname{conn}}\left(\Omega_{S_{0^{++}}}^{0} ; r_{2 \|}, \vec{r}_{2 \perp}\right) .
\end{aligned}
$$

Also in this case, the potential is attractive. ${ }^{12}$ For $N_{c}=3$, i.e., in quenched QCD, the mass of the lightest

\footnotetext{
${ }^{12}$ See footnote 9. In Appendix $\mathrm{D}$ we show that also $\mathcal{M}^{\text {conn }}\left(\Omega_{S_{0^{+}}}^{0} ; r_{\|}, \vec{r}_{\perp}\right)$ depends on $\vec{r}_{\perp}$ only through $\vec{r}_{\perp}^{2}$.
}

glueball is $m_{0^{++}} \simeq 1.73 \mathrm{GeV}$ [21], corresponding to an interaction range $m_{0^{++}}^{-1} \simeq 0.11 \mathrm{fm}$, so that the asymptotic regime should be reached at distances accessible to lattice calculations. In Fig. 2 we compare the functional dependence of Eq. (84) with the numerical results obtained on the lattice in Ref. [22]. The potential is determined from Wilson loops of length $T=8$ and width $\left|\vec{r}_{1,2}\right|=1$ in lattice units, on configurations obtained on a $16^{4}$ lattice at $\beta=6.0$, corresponding to lattice spacing $a \simeq 0.1 \mathrm{fm}$. Lattice results and analytical prediction are compatible, although within rather large numerical errors.

The most important subleading corrections come from the expansion in inverse powers of $b$ of the energy, the phase-space measure, and the matrix elements, keeping fixed the particle content, i.e., for two-pion states in QCD and for the lightest glueball state in pure-gauge theory. From Eqs. (75) and (76), and since terms linear in the momenta in the expansion of the matrix elements give vanishing contributions upon integration, we have that the first subleading term is of relative order $b^{-1}$.

From Eq. (77) we see that for a given particle content, with total number of particles $\mathcal{N}_{\alpha}$, the leading (in $b$ ) contribution comes from the irreducible pair of partitions with maximal $K+\bar{K}$, which cannot exceed $\mathcal{N}_{\alpha}+1$. In pure-gauge theory, where states with a nonvanishing one-particle matrix element are present, the maximal value is attained, e.g., by the pair of partitions where one is trivial (the whole set) and one is maximal (each element is a part). In QCD [and in similar theories with (pseudo-)Goldstone bosons] there are no such states, and nonvanishing matrix elements are at least of the two-particle type. As a consequence, one has $K, \bar{K} \leq\left[\mathcal{N}_{\alpha} / 2\right]$, so that $K+\bar{K} \leq \mathcal{N}_{\alpha}$ if $\mathcal{N}_{\alpha}$ is even and $K+\bar{K} \leq \mathcal{N}_{\alpha}-1$ if $\mathcal{N}_{\alpha}$ is odd. The leading contribution at the $\mathcal{N}_{\alpha}$-particle level is thus proportional to 


$$
\begin{aligned}
& \text { pure-gauge: } \frac{e^{-\mathcal{N}_{\alpha} m_{0^{+}} b}}{b^{\mathcal{N}_{\alpha}}}, \\
& \text { QCD }:\left.\frac{e^{-\mathcal{N}_{\alpha} m_{\pi} b}}{b^{\mathcal{N}_{\alpha}+\frac{1}{2}}}\right|_{\mathcal{N}_{\alpha} \text { even }},\left.\frac{e^{-\mathcal{N}_{\alpha} m_{\pi} b}}{b^{\mathcal{N}_{\alpha}+1}}\right|_{\mathcal{N}_{\alpha}>1, \text { odd }} .
\end{aligned}
$$

It is worth discussing briefly what happens in the presence of massless particles. In this case we expect the matrix elements to vanish as powers of the momenta for small $|\vec{p}|$ (see footnote 6). Here we drop the particle indices for simplicity. For a multiparticle state containing only such massless particles, we expect by symmetry that each of them contributes the same power, $\lambda$, of $|\vec{p}|$, to the small-momentum behavior of the matrix elements. Rather than rescaling the momenta as in Eq. (72), we now more conveniently set $b \vec{p}=\vec{q}$. For large $b$ we find that $V_{d d} \sim b^{-\gamma}$, with $\gamma=1+2 \lambda$ if one-particle matrix elements are nonzero, and $\gamma=3+4 \lambda$ if matrix elements are nonzero starting from the two-particle level. An explicit calculation shows that $\lambda=1$ for free photons, resulting in the well-known large-distance behavior of the dipoledipole electrostatic potential. ${ }^{13}$ If the same value is assumed for massless pions in the chiral limit, then we find $\gamma=7$, in agreement with Refs. [4,5].

\section{CONCLUSIONS}

In this paper we have derived a general nonperturbative formula for the asymptotic large-distance behavior of the potential between two static colorless dipoles, valid for a wide class of non-Abelian gauge theories, and for any dipole size. Our result is based only on the symmetries and on the nature of the spectrum of the relevant theories and is therefore a robust result. In particular, calculations involving any kind of approximation have to compare successfully to our predictions.

In the case of QCD, we have found the same dependence on the distance as in the results of Refs. [4,5], which are valid in the regime of small dipole sizes. We have also compared our results to the recent nonperturbative calculations of Refs. [6,7], which make use of the AdS/QCD approach and of the instanton liquid model, respectively. In both cases we find qualitative agreement with our results (apart from some "anomalies" which remain to be clarified).

We have also discussed the case of pure $S U\left(N_{c}\right)$ gauge theory, for which, to the best of our knowledge, there were so far no estimates, and compared our prediction with the available lattice results (for $N_{c}=3$ ) [22], finding agreement (within the rather limited accuracy of the numerical data).

We conclude by observing that the techniques developed in this paper could be easily generalized to the case of the correlator of two Euclidean Wilson loops forming a nonzero angle $\theta$, which is relevant to the study of soft high-energy scattering and hadronic total cross sections (see Ref. [17] and references therein).

\section{ACKNOWLEDGMENTS}

M. G. is supported by the Hungarian Academy of Sciences under "Lendület" Grant No. LP2011-011.

\section{APPENDIX A: DECOMPOSITION OF PAIRS OF PARTITIONS IN IRREDUCIBLE SUBPARTITIONS}

Let $S$ be a finite discrete set. We call irreducible a pair of partitions $\mathcal{A}_{K}(S)=\left\{a_{k}\right\}_{k=1, \ldots, K}$ and $\overline{\mathcal{A}}_{\bar{K}}(S)=\left\{\bar{a}_{\bar{k}}\right\}_{\bar{k}=1, \ldots, \bar{K}}$ of $S$, with $K$ and $\bar{K}$ parts, respectively, if there are no proper subsets $\mathcal{I}_{S} \subset I_{\mathcal{A}}=\{1, \ldots, K\}$ and $\overline{\mathcal{I}}_{S} \subset I_{\overline{\mathcal{A}}}=\{1, \ldots, \bar{K}\}$ such that $\cup_{k \in \mathcal{I}_{S}} a_{k}=\cup_{\bar{k} \in \overline{\mathcal{I}}_{S}} \bar{a}_{\bar{k}}$. An irreducible pair of partitions of $S$ will be denoted by $\left[\mathcal{A}_{K}, \overline{\mathcal{A}}_{\bar{K}}\right](S)$. We prove now the following statement:

Any pair of partitions $\mathcal{A}_{K}(S)$ and $\overline{\mathcal{A}}_{\bar{K}}(S)$ of a set $S$ can be written uniquely as a pair

$$
\mathcal{F}_{J}(S), \quad\left\{\left[\mathcal{A}_{K_{j}}, \overline{\mathcal{A}}_{\bar{K}_{j}}\right]\left(F_{j}\right)\right\}_{J},
$$

where $\mathcal{F}_{J}(S)=\left\{F_{j}\right\}_{j=1, \ldots, J}$ is a partition of $S$ in $J$ parts, and $\left[\mathcal{A}_{K_{j}}, \overline{\mathcal{A}}_{\bar{K}_{j}}\right]\left(F_{j}\right)$ are $J$ irreducible pairs of partitions of the disjoint sets $F_{j}$, with $\cup_{j=1}^{J} \mathcal{A}_{K_{j}}\left(F_{j}\right)=$ $\mathcal{A}_{K}(S)$ and $\cup_{j=1}^{J} \overline{\mathcal{A}}_{\bar{K}_{j}}\left(F_{j}\right)=\overline{\mathcal{A}}_{\bar{K}}(S)$.

Here the union of partitions of disjoint sets denotes the union of the corresponding families of sets. To prove this statement, notice that for any subset $S_{1} \subseteq S$ a partition $\mathcal{A}_{K}(S)$ provides a natural covering of $S_{1}$, defined as

$$
\mathcal{O}_{\mathcal{A}}\left[S_{1}\right]=\cup_{k=1}^{K}\left\{a_{k} \mid a_{k} \cap S_{1} \neq \emptyset\right\}
$$

The following properties of $\mathcal{O}_{\mathcal{A}}$ hold:

$$
\begin{array}{ll}
\text { 1. } S_{1} \subseteq \mathcal{O}_{\mathcal{A}}\left[S_{1}\right] ; & \text { 2. if } S_{1} \subseteq S_{2} \subseteq S, \\
\text { 3. } \mathcal{O}_{\mathcal{A}}\left[a_{k}\right]=a_{k} ; & \text { 4. if } S_{1}, S_{2} \subseteq S, \quad \text { then } \mathcal{O}_{\mathcal{A}}\left[S_{1}\right] \subseteq \mathcal{O}_{\mathcal{A}}\left[S_{2}\right]
\end{array}
$$

\footnotetext{
${ }^{13}$ Notice, however, that in this case our derivation of the cluster decomposition fails, since there is no gap in the spectrum of intermediate states.
} 
Fixed points $F=\mathcal{O}_{\mathcal{A}}[F]$ of $\mathcal{O}_{\mathcal{A}}$ coincide with their covering (self-covering), so they must be of the form $F=\cup_{k \in \mathcal{I}_{F}} a_{k}$ for some $\mathcal{I}_{F} \subseteq I_{\mathcal{A}}$. Consider next $\mathcal{O}_{\mathcal{A} \overline{\mathcal{A}}}[F] \equiv \mathcal{O}_{\mathcal{A}}\left[\mathcal{O}_{\overline{\mathcal{A}}}[F]\right]$. It is straightforward to show that $\mathcal{O}_{\mathcal{A} \overline{\mathcal{A}}}[F]=F$ if and only if $F$ is self-covering with respect to both $\mathcal{A}$ and $\overline{\mathcal{A}}$ (biself-covering); i.e., $F=\cup_{k \in \mathcal{I}_{F}} a_{k}=$ $\cup_{\bar{k} \in \overline{\mathcal{I}}_{F}} \bar{a}_{\bar{k}}$ for some $\mathcal{I}_{F} \subseteq I_{\mathcal{A}}$ and $\overline{\mathcal{I}}_{F} \subseteq I_{\overline{\mathcal{A}}}$. We call the partitions $\left\{a_{k}\right\}_{k \in \mathcal{I}_{F}}$ and $\left\{\bar{a}_{\bar{k}}\right\}_{\bar{k} \in \overline{\mathcal{I}}_{F}}$ the induced partitions of $F$. If the induced partitions of $F$ form an irreducible pair, then we say that $F$ is irreducible. By definition, an irreducible biself-covering set does not contain proper biself-covering subsets.

The proof now goes as follows. Since, by property 1 , $\mathcal{O}_{\mathcal{A} \overline{\mathcal{A}}}^{n}\left[a_{k}\right] \subseteq \mathcal{O}_{\mathcal{A} \overline{\mathcal{A}}}^{n+1}\left[a_{k}\right] \forall n \in \mathbb{N}$, and since $S$ is finite, there must be $n_{k} \in \mathbb{N}$ such that $F_{(k)} \equiv \mathcal{O}_{\mathcal{A} \overline{\mathcal{A}}}^{n_{k}}\left[a_{k}\right]=\mathcal{O}_{\mathcal{A} \overline{\mathcal{A}}}^{n_{k}+1}\left[a_{k}\right]=$ $\mathcal{O}_{\mathcal{A} \overline{\mathcal{A}}}\left[F_{(k)}\right]$; i.e., $F_{(k)}$ is biself-covering. We now show that the induced partitions of $F_{(k)}$ form an irreducible pair, so $F_{(k)}$ is irreducible. If not, there would be a biself-covering proper subset $F^{\prime} \subset F_{(k)}$, and since also $F^{\prime \prime}=F_{(k)} \backslash F^{\prime}$ would be biself-covering, we can assume without loss of generality that $a_{k} \subseteq F^{\prime}$. Then, by property 2 in Eq. (A2),

$$
F_{(k)}=\mathcal{O}_{\mathcal{A} \overline{\mathcal{A}}}^{n_{k}}\left[a_{k}\right] \subseteq \mathcal{O}_{\mathcal{A} \overline{\mathcal{A}}}^{n_{k}}\left[F^{\prime}\right]=F^{\prime} \subset F_{(k)},
$$

which is absurd. A similar argument shows that if $a_{k^{\prime}} \subseteq F_{(k)}$, then $F_{\left(k^{\prime}\right)}=F_{(k)}$, and analogously $\bar{F}_{(\bar{k})}=$ $F_{(k)}$ if $\bar{a}_{\bar{k}} \subseteq F_{(k)}$, with $\bar{F}_{(\bar{k})}$ generated from $\bar{a}_{\bar{k}}$ as described above. Finally, the sets $F_{(k)}$ are all the irreducible biselfcovering subsets of $S$ : if $F^{\prime}$ is an irreducible biself-covering set, then $\exists a_{k} \subseteq F^{\prime}$, and by property $2 F_{(k)} \subseteq F^{\prime}$, which contradicts irreducibility unless $F_{(k)}=F^{\prime}$. Obviously $\cup_{k} F_{(k)}=S$, and so the set $\left\{F_{j}\right\}_{j=1, \ldots, J}$ of the $J$ distinct $F_{(k)}$ 's provides the unique partition $\mathcal{F}_{J}(S)$ of $S$, such that the induced partitions of $F_{j}$, denoted by $\mathcal{A}_{K_{j}}\left(F_{j}\right)$ and $\overline{\mathcal{A}}_{\bar{K}_{j}}\left(F_{j}\right)$, form irreducible pairs $\left[\mathcal{A}_{K_{j}}, \overline{\mathcal{A}}_{\bar{K}_{j}}\right]\left(F_{j}\right)$. This completes the proof.

Obviously, to any pair $\mathcal{F}_{J}(S),\left\{\left[\mathcal{A}_{K_{j}}, \overline{\mathcal{A}}_{\bar{K}_{j}}\right]\left(F_{j}\right)\right\}_{J}$, with $\left[\mathcal{A}_{K_{j}}, \overline{\mathcal{A}}_{\bar{K}_{j}}\right]\left(F_{j}\right)$ any irreducible pair of partitions of $F_{j}$, corresponds a unique pair of partitions of $S$; i.e., $\mathcal{A}_{K}(S) \equiv$ $\cup_{j=1}^{J} \mathcal{A}_{K_{j}}\left(F_{j}\right)$ and $\overline{\mathcal{A}}_{\bar{K}}(S) \equiv \cup_{j=1}^{J} \overline{\mathcal{A}}_{\bar{K}_{j}}\left(F_{j}\right)$. The sum over pairs of partitions of a set $S$ can therefore be written equivalently as

$\sum_{K} \sum_{\mathcal{A}_{K}(S)} \sum_{\bar{K}} \sum_{\overline{\mathcal{A}}_{\bar{K}}(S)}=\sum_{J} \sum_{\mathcal{F}_{J}(S)} \prod_{F \in \mathcal{F}_{J}(S)}\left(\sum_{K} \sum_{\bar{K}} \sum_{\left[\mathcal{A}_{K}, \overline{\mathcal{A}}_{\bar{K}}\right](F)}\right)$.

Consider now the matrices

$$
A_{i}^{k}=\delta_{k \ell(i)}, \quad \bar{A}_{i}^{\bar{k}}=\delta_{\bar{k} \bar{\ell}(i)},
$$

where $\ell(i)$ and $\bar{\ell}(i)$ associate to each element $i \in S$ the labels of the parts of $\mathcal{A}_{K}$ and $\overline{\mathcal{A}}_{\bar{K}}$ that contain it. The columns $A^{k}$ and $\bar{A}^{\bar{k}}$ are not all linearly independent and satisfy exactly $J$ independent relations,

$$
\sum_{\left\{k \mid a_{k} \in F_{j}\right\}} A_{i}^{k}=\sum_{\left\{\bar{k} \mid \bar{a}_{\bar{k}} \in F_{j}\right\}} \bar{A}_{i}^{\bar{k}}, \quad j=1, \ldots, J .
$$

To see this, define the $J$ linear combinations

$$
Y_{i}^{(j)}(h, \bar{h}) \equiv \sum_{\left\{k \mid a_{k} \in F_{j}\right\}} A_{i}^{k} h_{k}-\sum_{\left\{\bar{k} \mid \bar{a}_{\bar{k}} \in F_{j}\right\}} \bar{A}_{i}^{\bar{k}} \bar{h}_{\bar{k}}
$$

which are immediately seen to be linearly independent, as they have no components in common. There are therefore at most $J$ relations of linear dependence among columns, of the form $Y_{i}^{(j)}(h, \bar{h})=0$, which in components read

$$
h_{\ell(i)}=\bar{h}_{\bar{\ell}(i)} \quad \forall i \in F_{j} .
$$

We now show that $u_{j}(i) \equiv h_{\ell(i)}=\bar{h}_{\bar{\ell}(i)}$ is constant over each $F_{j}$, from which Eq. (A6) follows. By definition, $h_{\ell(i)}$ is constant over any $a_{k}$, and similarly $\bar{h}_{\bar{\ell}(i)}$ is constant over any $\bar{a}_{\bar{k}}$, and so will be $u_{j}(i)$. Suppose now that $u_{j}(i)$ is constant over a subset $Q \subseteq F_{j}$. Then $u_{j}(i)$ is obviously constant in the covering of $Q$ provided by $\mathcal{A}_{K}$, since $\mathcal{O}_{\mathcal{A}}$ "completes" the parts already present in $Q$. By the same token, $u_{j}(i)$ will also be constant in $\mathcal{O}_{\overline{\mathcal{A}}}[Q]$ and in $\mathcal{O}_{\mathcal{A} \overline{\mathcal{A}}}[Q]$. Since $F_{j}=\mathcal{O}_{\mathcal{A} \overline{\mathcal{A}}}^{n_{k}}\left[a_{k}\right]$ for some $a_{k}$ and $n_{k} \in \mathbb{N}$, applying this argument repeatedly, we prove our statement.

As a final comment, consider the matrix obtained by adjoining the columns $\left\{k \mid a_{k} \in F_{j}\right\}$ of $A_{i}^{k}$ and $\left\{\bar{k} \mid \bar{a}_{\bar{k}} \in F_{j}\right\}$ of $\bar{A}_{i}^{\bar{k}}$. From the result above, its rank is $K_{j}+\bar{K}_{j}-1$. Since the rank has to be smaller than or equal to the number of rows, i.e., the total number of objects in $F_{j}, N_{j}$, we have $K_{j}+\bar{K}_{j}-1 \leq N_{j}$.

\section{APPENDIX B: EXPONENTIATION}

In this Appendix we discuss in some detail the derivation of the exponential formula, Eq. (66). In the previous Appendix, we have shown that each pair of partitions $\mathcal{A}_{K}\left(S_{\alpha}\right), \overline{\mathcal{A}}_{\bar{K}}\left(S_{\alpha}\right)$ of $S_{\alpha}$, with $K$ and $\bar{K}$ parts, respectively, can be uniquely rewritten as a partition $\mathcal{F}_{J}\left(S_{\alpha}\right)$ with $J$ parts and a set of irreducible pairs of partitions $\left[\mathcal{A}_{K_{j}}, \overline{\mathcal{A}}_{\bar{K}_{j}}\right]\left(F_{j}\right)$ of the parts $F_{j} \in \mathcal{F}_{J}\left(S_{\alpha}\right)$. Using Eq. (A4), and dropping temporarily the dependencies on $b, r_{1,2 \|}$, and $\vec{r}_{1,2 \perp}$ for simplicity, the product of two matrix elements $M^{(T)}\left(\Omega_{S_{\alpha}}\right)$ $\bar{M}^{(T)}\left(\Omega_{S_{\alpha}}\right)$ can be written as 


$$
M^{(T)}\left(\Omega_{S_{\alpha}}\right) \bar{M}^{(T)}\left(\Omega_{S_{\alpha}}\right)=\sum_{J} \sum_{\mathcal{F}_{J}\left(S_{\alpha}\right)} \prod_{F \in \mathcal{F}_{J}\left(S_{\alpha}\right)}\left\{\sum_{K} \sum_{\bar{K}} \sum_{\left[\mathcal{A}_{K}, \overline{\mathcal{A}}_{\bar{K}}\right](F)} \tilde{M}_{\left[\mathcal{A}_{K}, \overline{\mathcal{A}}_{\bar{K}}\right](F)}\left(\Omega_{F}\right)\right\},
$$

where

$$
\tilde{M}_{\left[\mathcal{A}_{K}, \overline{\mathcal{A}}_{\bar{K}}\right](F)}\left(\Omega_{F}\right) \equiv \prod_{a \in \mathcal{A}_{K}(F)} M^{(T) \operatorname{conn}}\left(\Omega_{a}\right) \prod_{\bar{a} \in \overline{\mathcal{A}}_{\bar{K}}(F)} \bar{M}^{(T) \operatorname{conn}}\left(\Omega_{\bar{a}}\right),
$$

and we have made us of the fact that, by construction,

$$
\bigcup_{a \in \mathcal{A}_{K}(F)} \Omega_{a}=\bigcup_{\bar{a} \in \overline{\mathcal{A}}_{\bar{K}}(F)} \Omega_{\bar{a}}=\Omega_{F} .
$$

A partition $\mathcal{F}_{J}\left(S_{\alpha}\right)$ of $S_{\alpha}$ in $J$ parts is fully specified by the $J$ strings $\alpha_{j} \equiv\left\{N_{s, j}\right\}$ of occupation numbers $N_{s, j}=N_{s}\left(\alpha_{j}\right)$ (the number of elements of type $s$ in part $j$ ), satisfying $\sum_{s} N_{s, j} \neq 0$ (while $N_{s, j}$ may be zero for some $s, j$ ) and $\sum_{j} N_{s, j}=N_{s}$, by a reference partition with the given occupation numbers, and by $n_{\mathrm{sp}}^{\alpha}$ permutations $P_{s} \in \mathcal{S}_{N_{s}}$, one for each of the $n_{\mathrm{sp}}^{\alpha}$ types that are present in $S_{\alpha}$. This representation is redundant, with $J ! \prod_{s, j} N_{s, j}$ ! pairs $\left(\left\{N_{s, j}\right\},\left\{P_{s}\right\}\right)$ corresponding to the same partition, since the labeling of the parts is irrelevant and permutations of elements of the same type within a part do not yield a new partition. The sum over partitions $\mathcal{F}_{J}\left(S_{\alpha}\right)$ can then be written explicitly as

$$
\sum_{\mathcal{F}_{J}\left(S_{\alpha}\right)}=\frac{1}{J !} \sum_{\left\{\alpha_{j}\right\}_{J}^{\alpha}} \prod_{j=1}^{J} \frac{1}{\prod_{s} N_{s}\left(\alpha_{j}\right) !} \sum_{\left\{P_{s} \in \mathcal{S}_{N_{s}}\right\}}, \quad \sum_{\left\{\alpha_{j}\right\}_{J}^{\alpha}} \equiv \sum_{\alpha_{1} \neq \varnothing} \ldots \sum_{\alpha_{J} \neq \varnothing} \prod_{s}\left[\delta_{\sum_{j^{\prime}=1}^{J} N_{s, j^{\prime}}, N_{s}}\right] .
$$

Consider now the phase-space integral of $M^{(T)}\left(\Omega_{S_{\alpha}}\right) \bar{M}^{(T)}\left(\Omega_{S_{\alpha}}\right)$. Since the integration measure is factorized, we have

$$
\left\langle\left\langle\prod_{F \in \mathcal{F}_{J}\left(S_{\alpha}\right)} \tilde{M}_{\left[\mathcal{A}_{K}, \overline{\mathcal{A}}_{\bar{K}}\right](F)}\left(\Omega_{F}\right)\right\rangle\right\rangle_{\Omega_{S_{\alpha}}}=\prod_{F \in \mathcal{F}_{J}\left(S_{\alpha}\right)}\left\langle\left\langle\tilde{M}_{\left[\mathcal{A}_{K}, \overline{\mathcal{A}}_{\bar{K}}\right](F)}\left(\Omega_{F}\right)\right\rangle\right\rangle_{\Omega_{F}},
$$

and so

$$
G_{S_{\alpha}}^{(T)}=\left\langle\left\langle M^{(T)}\left(\Omega_{S_{\alpha}}\right) \bar{M}^{(T)}\left(\Omega_{S_{\alpha}}\right)\right\rangle\right\rangle_{\Omega_{S_{\alpha}}}=\sum_{J} \sum_{\mathcal{F}_{J}\left(S_{\alpha}\right)} \prod_{F \in \mathcal{F}_{J}\left(S_{\alpha}\right)}\left\{\sum_{K} \sum_{\bar{K}} \sum_{\left[\mathcal{A}_{K}, \overline{\mathcal{A}}_{\bar{K}]}\right](F)}\left\langle\left\langle\tilde{M}_{\left[\mathcal{A}_{K}, \overline{\mathcal{A}}_{\bar{K}}\right](F)}\left(\Omega_{F}\right)\right\rangle_{\Omega_{F}}\right\} .\right.
$$

Taking into account that particles of the same type are indistinguishable, the sum over permutations in Eq. (B4) can be carried out trivially, and after a relabeling of the particles, we get

$$
\frac{G_{S_{\alpha}}^{(T)}}{\prod_{s} N_{s} !}=\sum_{J} \frac{1}{J !} \sum_{\left\{\alpha_{j}\right\}_{J}^{\alpha}} \prod_{j=1}^{J}\left\{\frac{1}{\prod_{s} N_{s}\left(\alpha_{j}\right) !} \mathcal{Q}_{\alpha_{j}}^{(T)}\right\}
$$

where

$$
\mathcal{Q}_{\alpha}^{(T)}=\sum_{K} \sum_{\bar{K}} \sum_{\left[\mathcal{A}_{K}, \overline{\mathcal{A}}_{\bar{K}}\right]\left(S_{\alpha}\right)}\left\langle\left\langle\tilde{M}_{\left[\mathcal{A}_{K}, \overline{\mathcal{A}}_{\bar{K}}\right]\left(S_{\alpha}\right)}\left(\Omega_{S_{\alpha}}\right)\right\rangle\right\rangle_{\Omega_{S_{\alpha}}}
$$

with the sum being over irreducible pairs of partitions only. Summing now over states with different particle content, and using standard combinatorics results, we finally obtain

$$
\mathcal{G}^{(T)}\left(b ; r_{1 \|}, \vec{r}_{1 \perp}, r_{2 \|}, \vec{r}_{2 \perp}\right)=\exp \left\{\sum_{\alpha \neq \emptyset} \frac{1}{\prod_{s} N_{s}(\alpha) !} \mathcal{Q}_{\alpha}^{(T)}\left(b ; r_{1 \|}, \vec{r}_{1 \perp}, r_{2 \|}, \vec{r}_{2 \perp}\right)\right\},
$$

where we have reinstated the full notation. 


\section{APPENDIX C: CONTRIBUTIONS TO THE POTENTIAL}

In this Appendix we compute the contributions $V_{\left[\mathcal{A}_{K}, \overline{\mathcal{A}}_{\bar{K}}\right]\left(S_{\alpha}\right)}$ to the static dipole-dipole potential, defined in Eq. (69). Recall that

$$
\begin{aligned}
-V_{\left[\mathcal{A}_{K}, \overline{\mathcal{A}}_{\bar{K}}\right]\left(S_{\alpha}\right)}\left(b ; r_{1 \|}, \vec{r}_{1 \perp}, r_{2 \|}, \vec{r}_{2 \perp}\right) \equiv & \lim _{T \rightarrow \infty} \frac{1}{T}\left\langle\left\langle\prod_{a \in \mathcal{A}_{K}\left(S_{\alpha}\right)} M^{(T) \operatorname{conn}}\left(\Omega_{a} ; r_{1 \|}, \vec{r}_{1 \perp}\right) \prod_{\bar{a} \in \overline{\mathcal{A}}_{\bar{K}}\left(S_{\alpha}\right)} \bar{M}^{(T) \operatorname{conn}}\left(\Omega_{\bar{a}} ; r_{2 \|}, \vec{r}_{2 \perp}\right)\right\rangle\right\rangle_{\Omega_{S_{\alpha}} ; b} \\
= & \lim _{T \rightarrow \infty} \frac{1}{T} \int d \Omega_{S_{\alpha}} e^{-b E\left(\Omega_{S_{\alpha}}\right)} \prod_{a \in \mathcal{A}_{K}\left(S_{\alpha}\right)} \int d t_{a} e^{i q_{a} t_{a}} F_{T}\left(t_{a}, P_{a} ; r_{1 \|}, \vec{r}_{1 \perp}\right) \\
& \times \prod_{\bar{a} \in \overline{\mathcal{A}}_{\bar{K}}\left(S_{\alpha}\right)} \int d \bar{t}_{\bar{a}} e^{-i \bar{q}_{\bar{a}} \overline{\bar{t}}_{\bar{a}}}\left[F_{T}\left(\bar{t}_{\bar{a}}, P_{\bar{a}} ; r_{2 \|},-\vec{r}_{2 \perp}\right)\right]^{*}
\end{aligned}
$$

where we have introduced the quantity

$$
F_{T}\left(t_{a}, P_{a} ; r_{\|}, \vec{r}_{\perp}\right) \equiv=\operatorname{Lim}_{a} \prod_{i_{s} \in a}\left[-i \pi^{(s)}\left(\vec{p}_{i_{s}}, s_{3 i_{s}}\right)\right]\left[\int d \hat{X}_{a} e^{i \mathcal{R}_{a}\left(\hat{X}_{a}, P_{a}\right)} C_{T}\left(t_{a}, \hat{X}_{a} ; r_{\|}, \vec{r}_{\perp}\right)\right]_{P_{a 4} \rightarrow e^{-i \frac{\pi}{2}}\left(-P_{a}^{0}\right)},
$$

where $d X_{a}=d t_{a} d \hat{X}_{a}$, and $P_{a} \cdot X_{a}=q_{a} t_{a}+\mathcal{R}_{a}\left(\hat{X}_{a}, P_{a}\right)$, with $q_{a}=\sum_{i_{s} \in a} p_{i_{s} 1}$, and $t_{a}=\frac{1}{N_{a}} \sum_{i_{s} \in a} x_{i_{s} 1}$ with $N_{a}$ the number of elements in $a$. The explicit form of the remainder $\mathcal{R}_{a}\left(\hat{X}_{a}, P_{a}\right)$ is not needed. Here we have dropped the subscript $E$ from Euclidean coordinates and momenta for simplicity. In terms of $F_{T}$, we have [see Eq. (61)]

$$
M^{(T) \operatorname{conn}}\left(\Omega_{a} ; r_{1 \|}, \vec{r}_{1 \perp}\right)=\int d t_{a} e^{i q_{a} t_{a}} F_{T}\left(t_{a}, P_{a} ; r_{1 \|}, \vec{r}_{1 \perp}\right)
$$

As discussed in Sec. III C, for large $T$

$$
\lim _{T \rightarrow \infty} F_{T}\left(T \tau_{a}, P_{a} ; r_{1 \|}, \vec{r}_{1 \perp}\right)=\chi\left(\tau_{a}\right) \mathcal{M}^{\mathrm{conn}}\left(\Omega_{a} ; r_{1 \|}, \vec{r}_{1 \perp}\right),
$$

with

$$
\mathcal{M}^{\operatorname{conn}}\left(\Omega_{a} ; r_{1 \|}, \vec{r}_{1 \perp}\right) \equiv \operatorname{Lim}_{a} \prod_{i_{s} \in a}\left[-i \pi^{(s)}\left(\vec{p}_{i_{s}}, s_{3 i_{s}}\right)\right]\left[\int d \hat{X}_{a} e^{i \mathcal{R}_{a}\left(\hat{X}_{a}, P_{a}\right)} C\left(\hat{X}_{a} ; r_{1 \|}, \vec{r}_{1 \perp}\right)\right]_{P_{a 4} \rightarrow e^{-i \frac{\pi}{2}}\left(-P_{a}^{0}\right)} .
$$

The other connected matrix element, $\bar{M}^{(T) \text { conn }}$ [see Eq. (60)], can be similarly recast as

$$
\bar{M}^{(T) \operatorname{conn}}\left(\Omega_{\bar{a}} ; r_{2 \|}, \vec{r}_{2 \perp}\right)=\int d \bar{t}_{\bar{a}} e^{-i \bar{q}_{\bar{a}} \bar{t}_{\bar{a}}}\left[F_{T}\left(\bar{t}_{\bar{a}}, P_{\bar{a}} ; r_{2 \|},-\vec{r}_{2 \perp}\right)\right]^{*}
$$

where $\bar{t}_{\bar{a}}=\frac{1}{N_{\bar{a}}} \sum_{i_{s} \in \bar{a}} x_{i_{s} 1}$ and $\bar{q}_{\bar{a}}=\sum_{i_{s} \in \bar{a}} p_{i_{s} 1}$. In full analogy with what was done above, we have

$$
\begin{aligned}
\lim _{T \rightarrow \infty}\left[F_{T}\left(T \bar{\tau}_{\bar{a}}, P_{\bar{a}} ; r_{2 \|},-\vec{r}_{2 \perp}\right)\right]^{*} & =\chi\left(\bar{\tau}_{\bar{a}}\right) \overline{\mathcal{M}}^{\mathrm{conn}}\left(\Omega_{\bar{a}} ; r_{2 \|}, \vec{r}_{2 \perp}\right), \\
\overline{\mathcal{M}}^{\mathrm{conn}}\left(\Omega_{\bar{a}} ; r_{2 \|}, \vec{r}_{2 \perp}\right) & =\left[\mathcal{M}^{\mathrm{conn}}\left(\Omega_{\bar{a}} ; r_{2 \|},-\vec{r}_{2 \perp}\right)\right]^{*} .
\end{aligned}
$$

To compute $V_{\left[\mathcal{A}_{K}, \overline{\mathcal{A}}_{\bar{K}}\right]\left(S_{\alpha}\right)}$, it is convenient to change variables and use $K+\bar{K}-1 \leq \mathcal{N}_{\alpha}$ out of the $K+\bar{K}$ linear combinations $q_{a}$ and $\bar{q}_{\bar{a}}$, which we denote collectively with $q$ and $\bar{q}$, and other $3 \mathcal{N}_{\alpha}-(K+\bar{K}-1)$ linearly independent combinations of the momenta, which we denote collectively with $\hat{P}$. That only $K+\bar{K}-1 \leq \mathcal{N}_{\alpha}$ of the $q_{a}$ and $\bar{q}_{\bar{a}}$ are independent follows from the results of Appendix A. Indeed, in the notation of Appendix A, $q=A p_{1}$ and $\bar{q}=\bar{A} p_{1}$, with $p_{1}$ denoting collectively all the $p_{i_{s}}$, and the matrix obtained by adjoining the columns of $A$ and $\bar{A}$ has $\operatorname{rank} K+\bar{K}-1$. 
The Jacobian of the change of variables can be chosen to be unity, and so we can write the phase-space integration measure as

$$
\int d \Omega_{S_{\alpha}}=\sum_{\left\{s_{3}\right\}} \int \prod_{i_{s} \in S_{\alpha}} \frac{d^{3} p_{i_{s}}}{(2 \pi)^{3}}=\int d \hat{P} \int d q \int d \bar{q} 2 \pi \delta\left(\sum_{a} q_{a}-\sum_{\bar{a}} \bar{q}_{\bar{a}}\right),
$$

where

$$
d q=\prod_{a \in \mathcal{A}_{K}} \frac{d q_{a}}{2 \pi}, \quad d \bar{q}=\prod_{\bar{a} \in \overline{\mathcal{A}}_{\bar{K}}} \frac{d \bar{q}_{\bar{a}}}{2 \pi}
$$

and $\int d \hat{P}$ is understood to include also the summation over spin, which plays no role in the following. We now set

$$
\mathcal{F}_{T}\left(t, \bar{t}, q, \bar{q}, \hat{P} ; b, r_{1 \|}, \vec{r}_{1 \perp}, r_{2 \|}, \vec{r}_{2 \perp}\right)=e^{-b E\left(\Omega_{S_{\alpha}}\right)} \prod_{a \in \mathcal{A}_{K}} F_{T}\left(t_{a}, P_{a} ; r_{1 \|}, \vec{r}_{1 \perp}\right) \prod_{\bar{a} \in \overline{\mathcal{A}}_{\bar{K}}}\left[F_{T}\left(\bar{t}_{\bar{a}}, P_{\bar{a}} ; r_{2 \|},-\vec{r}_{2 \perp}\right)\right]^{*}
$$

Dropping the dependence on $b$ and on size and orientation of the dipoles, $\mathcal{F}_{T}$ behaves as follows at large $T$ :

$$
\lim _{T \rightarrow \infty} \mathcal{F}_{T}(T \tau, T \bar{\tau}, q, \bar{q}, \hat{P})=\mathcal{F}(q, \bar{q}, \hat{P}) \prod_{a \in \mathcal{A}_{K}} \chi\left(\tau_{a}\right) \prod_{\bar{a} \in \overline{\mathcal{A}}_{\bar{K}}} \chi\left(\bar{\tau}_{\bar{a}}\right)
$$

With this notation, and using the integral representation of the Dirac delta, we can write

$$
-V_{\left[\mathcal{A}_{K}, \overline{\mathcal{A}}_{\overline{\mathcal{K}}}\right]\left(S_{\alpha}\right)}=\lim _{T \rightarrow \infty} \frac{1}{T} \int d \hat{P} \int d q \int d \bar{q} \int d \omega \int d t \int d \bar{t} e^{i\left[\sum_{a} q_{a}\left(t_{a}-\omega\right)-\sum_{\bar{a}} \bar{q}_{\bar{a}}\left(\bar{t}_{\bar{a}}-\omega\right)\right]} \mathcal{F}_{T}(t, \bar{t}, q, \bar{q}, \hat{P}) .
$$

Rescaling now $q_{a}, \bar{q}_{\bar{a}} \rightarrow q_{a} / T, \bar{q}_{\bar{a}} / T, t_{a}, \bar{t}_{\bar{a}} \rightarrow t_{a} T, \bar{t}_{\bar{a}} T$, and $\omega \rightarrow T \omega$, and using the large- $T$ behavior of $\mathcal{F}_{T}$, we find

$$
\begin{aligned}
-V_{\left[\mathcal{A}_{K}, \overline{\mathcal{A}}_{\bar{K}}\right]\left(S_{\alpha}\right)} & =\int d \hat{P} \int d q \int d \bar{q} \int d t \int d \bar{t} \int d \omega \mathcal{F}(0,0, \hat{P}) \prod_{a \in \mathcal{A}_{K}} \chi\left(t_{a}\right) e^{i q_{a}\left(t_{a}-\omega\right)} \prod_{\bar{a} \in \overline{\mathcal{A}}_{\bar{K}}} \chi\left(\bar{t}_{\bar{a}}\right) e^{-i \bar{q}_{\bar{q}}\left(\bar{t}_{\bar{a}}-\omega\right)} \\
& =\int d \hat{P} \int d \omega \mathcal{F}(0,0, \hat{P}) \chi(\omega)^{K+\bar{K}}=\int d \hat{P} \mathcal{F}(0,0, \hat{P}) .
\end{aligned}
$$

Changing integration variables back to the original ones, this expression can be recast in the following equivalent, but physically more clear form,

$$
\begin{aligned}
-V_{\left[\mathcal{A}_{K}, \overline{\mathcal{A}}_{\bar{K}}\right]\left(S_{\alpha}\right)}\left(b ; r_{1 \|}, \vec{r}_{1 \perp}, r_{2 \|}, \vec{r}_{2 \perp}\right) & =\int d \hat{P} \int d q \int d \bar{q} \mathcal{F}(q, \bar{q}, \hat{P}) 2 \pi \delta\left(\sum_{a} q_{a}\right) \prod_{a \in \mathcal{A}_{K}\left(S_{\alpha}\right)}^{\circ} 2 \pi \delta\left(q_{a}\right) \prod_{\bar{a} \in \overline{\mathcal{A}}_{\bar{K}}\left(S_{\alpha}\right)}^{\circ} 2 \pi \delta\left(\bar{q}_{\bar{a}}\right) \\
& =\int d \Omega_{S_{\alpha}} e^{-b E\left(\Omega_{S_{\alpha}}\right)}(2 \pi)^{K+\bar{K}-1} \delta_{\left[\mathcal{A}_{K}, \overline{\mathcal{A}}_{\bar{K}}\right]\left(S_{\alpha}\right)}\left(p_{1}\right) \tilde{M}_{\left[\mathcal{A}_{K}, \overline{\mathcal{A}}_{\bar{K}}\right]\left(S_{\alpha}\right)}\left(\Omega_{S_{\alpha}} ; r_{1 \|}, \vec{r}_{1 \perp}, r_{2 \|}, \vec{r}_{2 \perp}\right),
\end{aligned}
$$

where $\tilde{M}_{\left[\mathcal{A}_{K}, \overline{\mathcal{A}}_{\bar{K}}\right]\left(S_{\alpha}\right)}$ has been defined in Eq. (B2), and

$$
\delta_{\left[\mathcal{A}_{K}, \overline{\mathcal{A}}_{\bar{K}}\right]\left(S_{\alpha}\right)}\left(p_{1}\right) \equiv \delta\left(\sum_{i_{s} \in S_{\alpha}} p_{i_{s} 1}\right) \prod_{a \in \mathcal{A}_{K}\left(S_{\alpha}\right)}^{\circ} \delta\left(\sum_{i_{s} \in a} p_{i_{s} 1}\right) \prod_{\bar{a} \in \overline{\mathcal{A}}_{\bar{K}}\left(S_{\alpha}\right)}^{\circ} \delta\left(\sum_{i_{s} \in \bar{a}} p_{i_{s} 1}\right),
$$

where the symbol $\circ$ denotes that the product is over all the parts in the partition but one. 


\section{APPENDIX D: SELECTION RULES FOR SPIN-ZERO PARTICLES}

In this Appendix we derive the selection rule $\eta_{P}=\eta_{C}=1$ for the Wilson-loop matrix element corresponding to a state with a single, self-conjugate spin-zero particle. To this end, we first notice the following transformation laws for the Wilsonloop operator $\hat{\mathcal{W}}_{E}\left(r_{\|}, \vec{r}_{\perp} ; \hat{u}\right)$ under charge conjugation, $C$, and parity, $P$ [here we have made explicit also the dependence on the orientation of the long side, $\left.u_{E}=(1,0,0,0)=(\hat{u}, 0)\right]$ :

$$
\begin{aligned}
& \hat{U}(C) \hat{\mathcal{W}}_{E}\left(r_{\|}, \vec{r}_{\perp} ; \hat{u}\right) \hat{U}(C)^{\dagger}=\hat{\mathcal{W}}_{E}\left(r_{\|}, \vec{r}_{\perp} ;-\hat{u}\right)=\hat{\mathcal{W}}_{E}\left(-r_{\|},-\vec{r}_{\perp} ; \hat{u}\right), \\
& \hat{U}(P) \hat{\mathcal{W}}_{E}\left(r_{\|}, \vec{r}_{\perp} ; \hat{u}\right) \hat{U}(P)^{\dagger}=\hat{\mathcal{W}}_{E}\left(r_{\|},-\vec{r}_{\perp} ;-\hat{u}\right)=\hat{\mathcal{W}}_{E}\left(-r_{\|}, \vec{r}_{\perp} ; \hat{u}\right) .
\end{aligned}
$$

Notice that time is chosen again in the direction of the spatial separation $\vec{b}$ between the dipoles. Under rotations, $R$, one has in general $\hat{U}(R) \hat{\mathcal{W}}_{E}[\mathcal{C}] \hat{U}(R)^{\dagger}=\hat{\mathcal{W}}_{E}[R \mathcal{C}]$, with an obvious meaning of the notation. In particular, for $\hat{\mathcal{W}}_{E}\left(r_{\|}, \vec{r}_{\perp} ; \hat{u}\right)$ and for rotations of $\pi$ radians around the axes $\hat{r}_{\perp}$ and $\hat{u}$, denoted, respectively, by $R_{\perp}$ and $R_{u}$, we have

$$
\begin{aligned}
\hat{U}\left(R_{\perp}\right) \hat{\mathcal{W}}_{E}\left(r_{\|}, \vec{r}_{\perp} ; \hat{u}\right) \hat{U}\left(R_{\perp}\right)^{\dagger}=\hat{\mathcal{W}}_{E}\left(r_{\|}, \vec{r}_{\perp} ;-\hat{u}\right)=\hat{U}(C) \hat{\mathcal{W}}_{E}\left(r_{\|}, \vec{r}_{\perp} ; \hat{u}\right) \hat{U}(C)^{\dagger}, \\
\hat{U}\left(R_{u}\right) \hat{\mathcal{W}}_{E}\left(r_{\|}, \vec{r}_{\perp} ; \hat{u}\right) \hat{U}\left(R_{u}\right)^{\dagger}=\hat{\mathcal{W}}_{E}\left(r_{\|},-\vec{r}_{\perp} ; \hat{u}\right)=\hat{U}(P) \hat{U}(C) \hat{\mathcal{W}}_{E}\left(r_{\|}, \vec{r}_{\perp} ; \hat{u}\right) \hat{U}(C)^{\dagger} \hat{U}(P)^{\dagger} .
\end{aligned}
$$

For states $\left|\Omega_{S_{\alpha}}\right\rangle=|\vec{p}\rangle$ containing a single spin-zero particle, the relevant matrix element, $M\left(\vec{p} ; r_{\|}, \vec{r}_{\perp}\right)$, must be of the form

$$
M\left(\vec{p} ; r_{\|}, \vec{r}_{\perp}\right)=\delta(\vec{p} \cdot \hat{u}) f\left(\vec{p} \cdot \vec{r}_{\perp}, \vec{p}^{2} ; r_{\|}, \vec{r}_{\perp}^{2}\right),
$$

for some function $f$, as a consequence of rotation invariance, and of translation invariance along $\hat{u}$ in the limit $T \rightarrow \infty$. For a self-conjugate particle with parities $\eta_{C}$ and $\eta_{P}$, one has moreover, from Eq. (D2),

$$
\begin{aligned}
& M\left(\vec{p} ; r_{\|}, \vec{r}_{\perp}\right)=\eta_{C} M\left(R_{\perp} \vec{p} ; r_{\|}, \vec{r}_{\perp}\right)=\eta_{C} M\left(\vec{p} ; r_{\|}, \vec{r}_{\perp}\right), \\
& M\left(\vec{p} ; r_{\|}, \vec{r}_{\perp}\right)=\eta_{C} \eta_{P} M\left(-R_{u} \vec{p} ; r_{\|}, \vec{r}_{\perp}\right)=\eta_{C} \eta_{P} M\left(\vec{p} ; r_{\|}, \vec{r}_{\perp}\right),
\end{aligned}
$$

where Eq. (D3) was also used. The selection rules then follow immediately.

One can further exploit Lorentz invariance of the Minkowskian Wilson-loop matrix elements to prove that for spin-zero particles of mass $m$ the Euclidean matrix elements depend only on $r_{\|}$and $\vec{r}_{\perp}^{2}$ in the limit of vanishing spatial momentum. Indeed, for one-particle states,

$$
M_{M}\left(\vec{p} ; r_{\|}, \vec{r}_{\perp}\right)=\delta(\vec{p} \cdot \hat{u}) F_{M}\left(p \cdot R_{M}, R_{M}^{2}\right)=\delta(\vec{p} \cdot \hat{u}) F_{M}\left(p^{0} r_{\|}-\vec{p} \cdot \vec{r}_{\perp}, r_{\|}^{2}-\vec{r}_{\perp}^{2}\right),
$$

and after the Wick rotation $r_{\|} \rightarrow-i r_{\|}$,

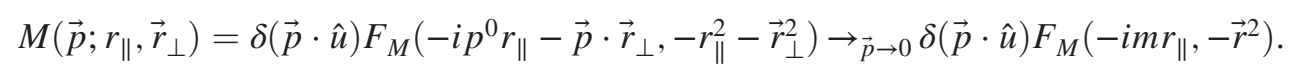

For two-particle states, $M\left(\vec{p}_{1}, \vec{p}_{2} ; r_{\|}, \vec{r}_{\perp}\right)$, one similarly has

$$
\begin{aligned}
& M_{M}\left(\vec{p}_{1}, \vec{p}_{2} ; r_{\|}, \vec{r}_{\perp}\right)=\delta\left(\left(\vec{p}_{1}+\vec{p}_{2}\right) \cdot \hat{u}\right) F_{M}\left(p_{1} \cdot p_{2}, p_{1} \cdot R_{M}, p_{2} \cdot R_{M}, p_{1} \cdot u_{M}, R_{M}^{2}\right), \\
& M\left(\vec{p}_{1}, \vec{p}_{2} ; r_{\|}, \vec{r}_{\perp}\right) \underset{\vec{p} \rightarrow 0}{\rightarrow} \delta\left(\left(\vec{p}_{1}+\vec{p}_{2}\right) \cdot \hat{u}\right) F_{M}\left(m^{2},-i m r_{\|},-i m r_{\|}, 0,-\vec{r}^{2}\right) .
\end{aligned}
$$

[1] T. Appelquist and W. Fischler, Phys. Lett. B 77, 405 (1978).

[2] G. Bhanot, W. Fischler, and S. Rudaz, Nucl. Phys. B155, 208 (1979).

[3] M. E. Peskin, Nucl. Phys. B156, 365 (1979).
[4] G. Bhanot and M. E. Peskin, Nucl. Phys. B156, 391 (1979).

[5] H. Fujii and D. Kharzeev, Phys. Rev. D 60, 114039 (1999).

[6] Y. Liu and I. Zahed, Phys. Rev. D 91, 055001 (2015).

[7] Y. Liu and I. Zahed, Phys. Rev. D 91, 034023 (2015). 
[8] L. S. Brown and W. I. Weisberger, Phys. Rev. D 20, 3239 (1979).

[9] H. Cheng and E. Tsai, Phys. Rev. D 36, 3196 (1987).

[10] H. Lehmann, K. Symanzik, and W. Zimmermann, Nuovo Cimento 1, 205 (1955).

[11] H. Lehmann, K. Symanzik, and W. Zimmermann, Nuovo Cimento 6, 319 (1957).

[12] V. S. Dotsenko and S. N. Vergeles, Nucl. Phys. B169, 527 (1980).

[13] R. A. Brandt, F. Neri, and M. Sato, Phys. Rev. D 24, 879 (1981).

[14] A. Di Giacomo, H. G. Dosch, V. I. Shevchenko, and Yu. A. Simonov, Phys. Rep. 372, 319 (2002).

[15] A. Di Giacomo, E. Meggiolaro, and H. Panagopoulos, Nucl. Phys. B483, 371 (1997).
[16] M. D’Elia, A. Di Giacomo, and E. Meggiolaro, Phys. Lett. B 408, 315 (1997).

[17] M. Giordano and E. Meggiolaro, J. High Energy Phys. 03 (2014) 002.

[18] B. Lucini, A. Rago, and E. Rinaldi, J. High Energy Phys. 08 (2010) 119.

[19] M. Kacir, M. Prakash, and I. Zahed, Acta Phys. Pol. B 30, 287 (1999).

[20] C. Gattringer, L. Y. Glozman, C. B. Lang, D. Mohler, and S. Prelovsek, Phys. Rev. D 78, 034501 (2008).

[21] C. J. Morningstar and M. J. Peardon, Phys. Rev. D 60, 034509 (1999).

[22] M. Giordano and E. Meggiolaro, Phys. Rev. D 81, 074022 (2010). 